\title{
Conjugação Quase-Simétrica em Sistemas Dinâmicos \\ Unidimensionais
}

\author{
Yong Su Han
}

Dissertação Apresentada

$\mathrm{aO}$

Instituto de Matemática e Estatística

da

Universidade de São Paulo

para Obtenção do Grau

de

Mestre em Matemática Aplicada

Área de Concentração: Sistemas Dinâmicos

Orientador: Prof. Dr. Edson Vargas

Durante a elaboração deste trabalho

o autor recebeu apoio financeiro do $\mathrm{CNPq}$

-São Paulo, Novembro de 1998 


\section{Conjugação Quase-Simétrica em Sistemas Dinâmicos Unidimensionais}

Este exemplar corresponde à redação final da dissertação devidamente corrigida e defendida por Yong Su Han e aprovada pela comissão ju!:

São Paulo, 14 de Janeiro de 1999.

Banca Examinadora:

-Prof. Dr. Edson Vargas (Orientador) - IME-USP

-Prof. Dr. Edson de Faria

- IME-USP

-Prof. Dr. Wellington de Melo

- IMPA 
a Teresa, minha idônea auxiliadora em Cristo. 


\section{Abstract}

On this work we study conjugacies between $C^{2}$ unimodal maps and tent maps. We exhibit a topological condition that prohibits the existence of a quasi-simmetric conjugacy between a $C^{2}$ unimodal map and a tent map. We also prove that almost every tent map satisfies it. This fact depends on the density of the critical orbit of a tent map which is true for almost all parameters. Our main tools are the concept of kneading map and köbe principles. 


\section{Agradecimentos}

Dou graças a Deus que me tem ajudado a estar neste momento da realização desta dissertação. Que só Ele seja louvado por este trabalho.

Agradeço de coração ao Prof. Edson Vargas pela sua sugestão do assunto deste trabalho e inestimável orientação nos momentos importantes, a ele sou profundamente grato.

Quero agradecer também aos demais professores do Instituto, que contribuíram para meu amadurecimento matemático. Em especial, ao Prof. Eduardo A. Prado e ao Prof. Luiz Fishman pelo incentivo no estudo da área de Sistemas Dinâmicos.

Ao colega Munhoz, agradeço pelo companheirismo e palavras de auxílio e estímulo.

Aos colegas da igreja UBF, sou profundamente agradecido pela oração e palavras de carinho em todos os momentos. 


\section{Sumário}

1 Conceitos Básicos 1

1.1 Funções absolutamente contínuas . . . . . . . . . . . . . . . . . 1

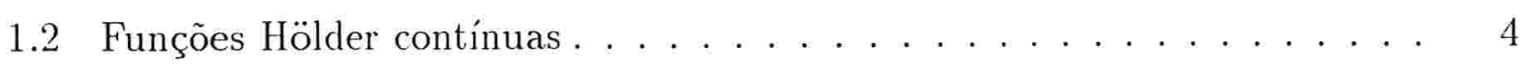

1.3 Homeomorfismos quase-simétricos . . . . . . . . . . . . . 6

1.4 Vários tipos de homeomorfismos . . . . . . . . . . . . . 7

1.5 Exemplos . . . . . . . . . . . . . . . . . . . 9

2 Dinâmica de Funções Unimodais 17

2.1 Conceitos básicos . . . . . . . . . . . . . . . . . 17

2.2 Conjugação quase-simétrica . . . . . . . . . . . . . . 20

3 Principais Instrumentos $\quad 24$

3.1 Razão cruzada . . . . . . . . . . . . . . . . . . . . . . . . . . 24

3.2 Princípios de Köbe . . . . . . . . . . . . . . . . . . . . . . . . . . . . . . 29

3.3 Aplicação kneading . . . . . . . . . . . . . . . . 35

4 Órbita Crítica da Função Tenda 40

4.1 Aplicação kneading e a órbita crítica . . . . . . . . . . . . . 40

4.2 Dependência do parâmetro . . . . . . . . . . . . . . . . 42

4.3 Intervalos de monotonicidade . . . . . . . . . . . . . . . . . . 47 
5 Conjugação Quase-simétrica

5.1 Uma condição topológica . . . . . . . . . . . . . . . . . . 51

6 Prova dos Principais Resultados $\quad 57$ 


\section{Introdução}

Um sistema dinâmico unidimensional é gerado por uma função contínua $f: I \rightarrow I$, onde $I$ é um intervalo compacto chamado espaço de fase. Este sistema involve infinitas funções, as iteradas de $f$, definidas indutivamente por $f^{1}=f$ e $f^{n+1}=f \circ f^{n}$. Paralelamente, no espaço de fase temos as noções de órbita positiva, órbita negativa e grande órbita de um ponto $x$ definidas por $\left\{y \in I ; f^{n}(x)=y, n \geq 0\right\},\left\{y \in I ; f^{n}(y)=x, n \geq 0\right\}$ e $\left\{y \in I ; f^{m}(y)=f^{n}(x), m, n \geq 0\right\}$ respectivamente.

Na teoria qualitativa dos sistemas dinâmicos a relação de equivalência natural para expressar a noção de "mesma dinâmica" é o que chamamos conjugação, a saber: duas funções $f$ e $g$ são conjugadas se existe um homeomorfismo $h: I \rightarrow I$ tal que $h \circ f=$ $g \circ h$. Um tal homeomorfismo, chamado uma conjugação entre $f$ e $g$, aplica órbitas de $f$ em órbitas de $g$ e preserva propriedades topológicas do sistema. No entanto também temos interesse em propriedades métricas e geométricas do sistema e entào necessitamos que as conjugações tenham mais regularidade, a saber: que sejam ou analíticas, ou diferenciáveis, ou quase-simétricas, ou Hölder contínuas, ou absolutamente contínuas.

Neste trabalho estamos interessados em sistemas dinâmicos gerados por iterações de funções unimodais, veja definição adiante. As funções quadráticas $q_{a}:[-1,1] \rightarrow[-1,1]$ dadas por $q_{a}(x)=-1+a\left(1-x^{2}\right)$ e as funções tendas $T_{a}: I_{a} \rightarrow I_{a}$ dadas por $T_{a}(x)=$ $1-a|x|$ com $a \in[1,2]$, onde $I_{a}=\left[\frac{1}{1-a}, \frac{1}{a-1}\right]$, são importantes exemplos de funções unimodais. 
Milnor e Thurston provaram que uma função unimodal $f$ com entropia topológica positiva $h_{\text {top }}(f)$ é semi-conjugada a uma função tenda $T_{a} \operatorname{com} a=\exp \left(h_{\text {top }}(f)\right)$. Muitas vezes esta semi-conjugação é de fato uma conjugação. Neste trabalho estudamos a regularidade desta conjugação (quando existir) no caso em que $f$ é de classe $C^{2}$. Nesta situação a referida conjugação nunca é um difeomorfismo. Por outro lado, segue de $[\mathrm{NP}]$ que existe um conjunto de parâmetros com medidade de Lebesgue positiva tal que as correspondentes funções tendas são Hölder continuamente conjugadas a funções unimodais de classe $C^{2}$. Examinamos então com que freqüência uma função tenda $T_{a}$ é quase-simetricamente conjugada a uma função unimodal de classe $C^{2}$. Isto depende de propriedades topológicas como a densidade ou não da órbita positiva do ponto crítico. Baseados nisto exibiremos uma condição que proibe a existência de uma conjugação quase-simétrica entre uma função tenda e uma função unimodal de classe $C^{2}$. Esta condição é satisfeita por funções tendas $T_{a} \operatorname{com} a$ em um conjunto com medida de Lebesgue total em $[1,2]$ resultando então o fato de que uma função tenda raramente é quase-simetricamente conjugada a uma função unimodal de classe $C^{2}$. Isto contrasta com a situação de funções expansivas do círculo de um dado grau e classe $C^{1+\alpha}$, as quais são sempre quase-simetricamente conjugadas entre si. No caso de difeomorfismos do círculo de classe $C^{3}$ cujo número de rotação é irracional e Diophantino são $C^{1}$-conjugadas à uma rotação, veja [YJ]. Enfatizamos o fato de que a inversa de um homeomorfismo quase-simétrico também é quase-simétrica, portanto conjugação quase-simétrica é uma relação reflexiva.

No capítulo 1 estudamos vários conceitos importantes em dinâmica tais como: diferenciabilidade, quase-simetria, Hölder continuidade e continuidade absoluta. São dados exemplos e provados alguns resultados que mostram a relação entre estas classes de homeomorfismos. No capítulo 2 estudamos alguns aspectos da dinâmica das funções unimodais e exibimos uma propriedade que é satisfeita pelas funções unimodais que são quase-simetricamente conjugadas a funções tendas. No capítulo 3 provamos os Princípios de Köbe, inclusive no caso em que as funções unimodais sào de classe $C^{2}$, que nos permite fazer estimativas da distorção das iteradas destas funçòes. E, ainda no mesmo capítulo, 
estudamos o conceito de aplicação kneading que será utilizado no estudo da densidade ou não da órbita crítica de uma função tenda. No capítulo 4 estudamos a densidade da órbita crítica de funções tendas. No capítulo 5 exibimos uma condição topológica que proibe a existência de uma conjugação quase-simétrica entre uma função unimodal de classe $C^{2}$ e uma função tenda. Mostramos também que essa condição é satisfeita por quase todas as funções tendas (um conjunto de parâmetros de medida de Lebesgue total em $[1,2])$. Este trabalho é baseado principalmente nas seguintes referências: [BD], [BH], [HF] e [MS]. 


\section{Principais Resultados}

Definição 0.0.1 Dado um intervalo I compacto, uma funçào contínua $f: I \rightarrow I$ é chamada unimodal se possui um único máximo local assumido em um ponto c no interior de $I$ e $f(\partial I) \subset \partial I$.

Observamos que se uma função unimodal é diferenciável então o seu único máximo local é um ponto crítico. Convencionamos chamar o ponto $c$ de ponto crítico mesmo no caso em que a função unimodal em questão não seja diferenciável.

Exemplo 0.0.1 (Funções tendas) As funçôes tendas $T_{a}: I_{a} \rightarrow I_{a}$ definidas por $T_{a}(x)=1-a|x|$ com $a \dot{\epsilon}(1,2]$ e $I_{a}:=\left[\frac{1}{1-a}, \frac{1}{a-1}\right]$, são funçôes unimodais cujo máximo é assumido em $c=0$. Observamos que $T_{a}$ não é diferenciável em $c=0$.

Exemplo 0.0.2 (Funções quadráticas) As funções quadráticas $q_{a}:[-1,1] \rightarrow[-1,1]$ definidas por $q_{a}(x)=-1+a\left(1-x^{2}\right)$ com $a \in(0,2]$ são funçôes unimodais cujo máximo é assumido em $c=0$.

\section{Outros Exemplos}

1. As funções $f_{a}:[0,1] \rightarrow[0,1]$ definidas por $f_{a}(x)=a \sin \pi x$ com $a \in(0,1]$ são funções unimodais cujo valor máximo é assumido em $c=1 / 2$.

2. As funções $P_{a}:[-1,1] \rightarrow[-1,1]$ definidas por $P_{a}(x)=-1+a\left(1-x^{2 l}\right)$ com $l \geq 1$ e $a \in(0,2]$ são funções unimodais cujo máximo é assumido em $c=0$. 
O comportamento da órbita positiva do ponto crítico de uma funçào unimodal desempenha papel essencial no entendimento de vários aspectos do sistema dinâmico correspondente. No caso de uma funçào tenda $T_{a}$ temos que freqüentemente esta órbita é densa em um intervalo, isto é o conteúdo do seguinte teorema (veja [BD]).

Teorema A Existe um conjunto $A \subset[\sqrt{2}, 2]$ com medida de Lebesgue total tal que para todo a $\in$ A verifica-se que a órbita crítica positiva da funçâo tenda $T_{a}$ é densa no intervalo $\left[T_{a}^{2}(c), T_{a}(c)\right]$.

Este teorema é uma importante etapa em direção à verificação do fato de que raramente uma função tenda $T_{a}$ é quase-simetricamente conjugada a uma função unimodal de classe $C^{2}$. Este fato é o conteúdo do seguinte teorema (veja $[\mathrm{BH}]$ ).

Teorema B. Existe um conjunto $A \subset[\sqrt{2}, 2]$ com medida de Lebesgue total tal que para $a \in A$ verifica-se que a funçâo tenda $T_{a}$ não é quase-simetricamente conjugada a uma função unimodal de classe $C^{2}$.

A prova do Teorema A depende do comportamento dos pontos da órbita crítica positiva de $T_{a}$ em relação ao parâmetro. A prova do Teorema $\mathrm{B}$ depende do conceito de aplicação kneading e dos princípios de liöbe os quais são importantes ferramentas em dinâmica unidimensional. 


\section{Capítulo 1}

\section{Conceitos Básicos}

Neste capítulo apresentamos algumas classes de homeomorfismos que surgem como conjugações entre dois sistemas dinâmicos. Estamos principalmente interessados em dinâmica unidimensional e sendo assim estudaremos as classes dos homeomorfismos do intervalo $[-1,1]$ que são, ou diferenciáveis, ou quase-simétricos, ou Hölder contínuos, ou absolutamente contínuos.

\subsection{Funções absolutamente contínuas}

Definição 1.1.1 Uma função $h:[a, b] \rightarrow \mathbf{R}$, é dita absolutamente contínua, se para todo $\epsilon>0$ existir $\delta>0$ tal que

$$
\sum_{i=1}^{n}\left|h\left(\beta_{i}\right)-h\left(\alpha_{i}\right)\right|<\epsilon
$$

para qualquer $n$ finito ou infinito e qualquer coleçào de intervalos disjuntos $\left(\alpha_{1} . \beta_{1}\right), \cdots,\left(\alpha_{n}, \beta_{n}\right)$ que satisfaz $\sum_{i=1}^{n}\left(\beta_{i}-\alpha_{i}\right)<\delta$.

Observamos que se $h$ é absolutamente contínua, então $h$ é em particular contínua. De fato, basta tomarmos $n=1$ na definição acima. 
Neste trabalho denotamos a $\sigma$-álgebra dos Borelianos de um intervalo $[a, b]$ por $\mathcal{B}$ e a respectiva medida de Lebesgue por $\lambda$. Dada uma medida $\mu$ sobre $\mathcal{B}$ e $p>0$ denotamos por $L^{p}(\mu)$ o conjunto das funçòes $h:[a, b] \rightarrow \mathbf{R}$ que são mensuráveis e tais que $\int_{[a, b]}|h|^{p} d \mu<$ $\infty$.

Dadas duas medidas $\mu$ e $v$ sobre $\mathcal{B}$ dizemos que $\mu$ é absolutamente contínua com respeito a $v($ denotamos $\mu \ll v)$ se para todo $E$ em $\mathcal{B}$ com $v(E)=0$ também temos $\mu(E)=0$. O teorema de Radon-Nikodym enunciado a seguir estabelece uma relação entre funções $L^{1}$ e medidas absolutamente contínuas com respeito a medida de Lebesgue. Este fato desempenha um importante papel para provar a relação equivalente entre a continuidade absoluta e a diferenciabilidade Lebesgue q.t.p.

Teorema 1.1.1 (Teorema de Radon-Nikodym) Dada uma medida $\mu$ sobre $\mathcal{B}$ são equivalentes:

1. A medida $\mu$ é absolutamente contínua com respeito a medida de Lebesgue $\lambda$.

D. Existe uma função $h:=\frac{d \mu}{d \lambda} \in L^{1}(\lambda)$ tal que

$$
\mu(E)=\int_{E} h d \lambda .
$$

Prova: Veja [WR].

Teorema 1.1.2 Suponha que $\mu$ e $v$ sejam medidas sobre uma $\sigma$-álgebra $\mathcal{A}$, entĩo são equivalentes:

1. A medida $\mu$ é absolutamente contínua com respeito à medida $v$.

2. Para todo $\epsilon>0$ existe $\delta>0$ tal que para todo $E \in \mathcal{A} \operatorname{com} v(E)<\delta$ tem-se $\mu(E)<\epsilon$

Prova: Veja [WR]. 
Teorema 1.1.3 Seja $h:[a, b] \rightarrow \mathbf{R}$ uma função contínua $\epsilon$ não-decrescente. Então são equivalentes:

1. A função $h$ é absolutamente contínua.

2. A função h aplica conjuntos de medida de Lebesgue zero em conjuntos de medida de Lebesgue zero.

3. A função $h$ é diferenciável em um conjunto com medida de Lebesgue total, a derivada $h^{\prime} \in L^{1}(\lambda) e$

$$
h(x)-h(a)=\int_{[a, x]} h^{\prime} d \lambda .
$$

Prova: 1) $\rightarrow$ 2) Assumimos que $h$ é absolutamente contínua em $[a, b]$ e consideramos $E \in \mathcal{B} \operatorname{com} \lambda(E)=0$. Dado $\epsilon>0$ consideramos também a constante $\delta>0$ correspondente dada pela Definição 1.1.1. Existe então um conjunto aberto em $[a, b]$ o qual denotamos por $V$ tal que $E \subset V$ e $\lambda(V)<\delta$. Seja $\left\{\left(\alpha_{i}, \beta_{i}\right)\right\}_{i=1}^{n}$ (n finito ou infinito) uma coleção (finita ou enumerável) de intervalos disjuntos cuja união é $V$. Então $\sum_{i=1}^{n}\left(\beta_{i}-\alpha_{i}\right)<\delta$ e pela escolha de $\delta$ temos que $\sum_{i=1}^{n}\left|h\left(\beta_{i}\right)-h\left(\alpha_{i}\right)\right| \leq \epsilon$. Como $E \subset \bigcup_{i=1}^{n}\left(\alpha_{i}, \beta_{i}\right)$ resulta que $\lambda(h(E)) \leq \epsilon$. Como $\epsilon>0$ é arbitrário e a $\sigma$-álgebra dos Borelianos munida da medida de Lebesgue forma um espaço de medida completo segue-se que $\lambda(h(E))=0$.

2) $\rightarrow$ 3) Definimos a função $g:[a, b] \rightarrow \mathbf{R}$ por $g(x)=x+h(x)$. Se a $h$-imagem de um intervalo de comprimento $\eta$ tem comprimento $\eta^{\prime}$, então a $g$-imagem do mesmo intervalo tem comprimento $\eta+\eta^{\prime}$. Deste fato, segue que $g$ satisfaz 2. Dado $E \in \mathcal{B}$ escrevemos $E=E_{1} \cup E_{0}$, onde $\lambda\left(E_{0}\right)=0$ e $E_{1}$ é uma união enumerável de conjuntos compactos, sendo $g$ contínua resulta que $g\left(E_{1}\right)$ também é uma união enumerável de conjuntos compactos e $g\left(E_{1}\right) \in \mathcal{B}$. Como $g$ satisfaz 2 temos que $\lambda\left(g\left(E_{0}\right)\right)=0$. E como $g(E)=g\left(E_{1}\right) \cup g\left(E_{0}\right)$, isto implica que $g(E) \in \mathcal{B}$. Então definimos

$$
\mu(E)=\lambda(g(E)), E \in \mathcal{B}
$$


Como $g$ é injetora ela aplica conjuntos disjuntos em conjutos disjuntos. A $\sigma$ - aditividade de $\lambda$ implica que $\mu$ é uma medida em $\mathcal{B}$. Também temos que $\mu \ll \lambda$ uma vez que $g$ satisfaz 2. Então, pelo Teorema de Radon-Nikodym existe $\xi \in L^{1}(\lambda)$ tal que $d \mu=\xi d \lambda$. Se $E=[a, x]$, então $g(E)=[g(a), g(x)] \mathrm{e}$

$$
g(x)-g(a)=\lambda(g(E))=\mu(E)=\int_{E} \xi d \lambda .
$$

Da definição de $g$ resulta que

$$
h(x)-h(a)=\int_{[a, x]}(\xi-1) d \lambda .
$$

Portanto $h^{\prime}(x)=\xi(x)-1$ Lebesgue q.t.p.

$3) \rightarrow 1$ ) Como $h^{\prime} \in L^{1}(\lambda)$, então existe uma medida $\mu$ definida por $d \mu=h^{\prime} d \lambda$. Como $\mu \ll \lambda$, pelo Teorema 1.1.2, para todo $\epsilon>0$ existe $\delta>0$ tal que $\mu(E)<\epsilon$ sempre que $\lambda(E)<\delta$. E, particularmente, no caso em que $E$ é união de intervalos disjuntos $\left\{\left(\alpha_{i}, \beta_{i}\right)\right\}_{i=1}^{n}$ tal que $\cup_{i=1}^{n}\left(\alpha_{i}, \beta_{i}\right)$, temos que $\sum_{i=1}^{n}\left|h\left(\alpha_{i}\right)-h\left(\beta_{i}\right)\right|<\epsilon$. Disto resulta que $h$ é absolutamente contínua.

\subsection{Funções Hölder contínuas}

Definição 1.2.1 Uma função $h:[a, b] \rightarrow \mathbf{R}$ é dita $(\alpha, M)$-Hölder contínua, se para todo $x, y \in[a, b]$ verifica-se que

$$
|h(x)-h(y)| \leq M|x-y|^{\alpha} .
$$

Então $h$ é dita Hölder contínua se for $(\alpha, M)$-Hölder contínua para algum $(\alpha, M)$. Em particular, quando $\alpha=1$, dizemos que $h$ é $M$-Lipschtziana. Dizemos que $h$ é Lipschtziana se for $M$-Lipschtziana para algum $M$. 
Lema 1.2.1 Considere uma função $h:[a, b] \rightarrow \mathbf{R}$, então temos que:

1. Se hé de classe $C^{1}$ entâo hé Lipschtziana.

2. Se $h$ é $(\alpha, M)$-Hölder contínua com $\alpha>1$ então $h$ é constante.

3. Se $h \in L^{p}(\lambda)$ entào $H(x):=\int_{[a, x]} h d \lambda$ é $\left(\frac{1}{q}, M\right)$-Hölder contínua, onde $\frac{1}{p}+\frac{1}{q}=1$ $\epsilon M:=\left(\int_{[a, b]}|h|^{p} d \lambda\right)^{1 / p}$.

Prova: 1) Se $h$ é de classe $C^{1}$ então para $x, y \in[a, b]$ temos, pelo teorema do valor médio, que existe $x_{o} \in(x, y)$ tal que

$$
|h(x)-h(y)|=\left|h^{\prime}\left(x_{0}\right)\right||x-y| .
$$

Segue então que $h$ é $M$-Lipschtziana para $M:=\sup \left\{\left|h^{\prime}(x)\right| ; x \in[a, b]\right\}$.

2) Se $\alpha>1$ então para $x \in[a, b]$ temos que

$$
\frac{|h(x+\delta)-h(x)|}{\delta} \leq M|\delta|^{\alpha-1} .
$$

Disto resulta que $h$ é derivável e $h^{\prime}(x)=0$ para todo $x \in[a, b]$. Portanto $h$ é constante.

3) Para todo $x, y \in[a, b]$ temos que

$$
|H(x)-H(y)|=\left|\int_{[y, x]} h d \lambda\right|
$$

e da desigualdade de Hölder concluímos que

$$
|H(x)-H(y)| \leq\left(\int_{[y, x]}|h|^{p} d \lambda\right)^{1 / p}\left(\int_{[y, x]}|1|^{q} d \lambda\right)^{1 / q} \leq M|x-y|^{1 / q},
$$

concluindo a prova tomamos $M:=\left(\int_{[a, b]}|h|^{p} d \lambda\right)^{1 / p}$ 


\subsection{Homeomorfismos quase-simétricos}

Definição 1.3.1 Dado $K \geq 1$, um homeomorfismo $h:[a, b] \rightarrow \mathbf{R}$ é dito $K$-quasesimétrico, se para todo $x \in[a, b]$ e $\epsilon>0$ tais que $a \leq x-\epsilon<x+\epsilon \leq b$ verifica-se que

$$
\frac{1}{K} \leq \frac{|h(x+\epsilon)-h(x)|}{|h(x)-h(x-\epsilon)|} \leq K^{*}
$$

Dizemos que $h$ é quase-simétrico se for $K$-quase-simétrico para algum $K$.

Lema 1.3.1 Se $h:[a, b] \rightarrow[c, d]$ é um homeomorfismo quase-simétrico, entào para todo $A>0$ existe $B>0$ tal que, se $J_{1}$ e $J_{2}$ são intervalos adjacentes contidos em $[a, b]$ com $\left|J_{2}\right| \leq A\left|J_{1} \cup J_{2}\right|$, verifica-se que $\left|h\left(J_{2}\right)\right| \leq B\left|h\left(J_{1} \cup J_{2}\right)\right|$.

Prova: Sejam $J_{1}=(x, v)$ e $J_{2}=(v, y)$, onde $x<v<y$. Denifimos $\omega_{n} \in J_{1} \cup J_{2}$ indutivamente tomamos $\omega_{0}=x$ e em geral $\omega_{n+1}=\frac{\omega_{n}+y}{2}$. A seqüência $\left\{\omega_{n}\right\}_{n=0}^{\infty}$ assim definida é crescente e converge para $y$ quando $n$ tende a infinito. Portanto existe $N$ tal que $\left|y-\omega_{N+1}\right| \leq A\left|x-\omega_{N+1}\right|$ e $\omega_{N}<v \leq \omega_{N+1}$. Como $h$ é quase-simétrico, para todo $i \geq 1$ temos que

$$
\frac{\left|h\left(\omega_{i}\right)-h\left(\omega_{i-1}\right)\right|}{\left|h(y)-h\left(\omega_{i}\right)\right|} \geq \frac{1}{K}
$$

e

$$
\frac{\left|h\left(\omega_{i-1}\right)-h(y)\right|}{\left|h\left(\omega_{i}\right)-h(y)\right|}=\frac{\left|h\left(\omega_{i-1}\right)-h\left(\omega_{i}\right)\right|+\left|h\left(\omega_{i}\right)-h(y)\right|}{\left|h\left(\omega_{i}\right)-h(y)\right|} \geq \frac{K+1}{K} .
$$

Portanto

$$
\left|h\left(\omega_{i}\right)-h(y)\right| \leq \frac{K}{1+K}\left|h\left(\omega_{i-1}\right)-h(y)\right|
$$

e então,

$$
\begin{aligned}
\left|h\left(J_{2}\right)\right| & \leq\left|\dot{h}(y)-h\left(\omega_{N}\right)\right| \leq\left(\frac{K}{1+K}\right)^{N}|h(y)-h(x)| \\
& =\left(\frac{K}{1+K}\right)^{N}\left|h\left(J_{1} \cup J_{2}\right)\right| .
\end{aligned}
$$

Tomamos então $B=\left(\frac{K}{1+K}\right)^{N}$. 
Do Lema anterior sabemos que quando $A$ tende a zero, então $N$ tende a infinito e isto resulta que $B$ também tende a zero. Deste fato temos seguinte corolário.

Corolário 1.3.1 Seja $h:[a, b] \rightarrow[c, d]$ um homeomorfismo quase-simétrico. E, dado um ponto $x \in[a, b]$, sejam $\left\{z_{k}\right\}_{k=1}^{\infty},\left\{y_{k}\right\}_{k=1}^{\infty}$ seqüencias em $[a, b]$ com $z_{k}<y_{k}<x$ ou $x<y_{k}<z_{k}$ para todo $k$. Então, se $\lim _{k \rightarrow \infty} \frac{\left|z_{k}-y_{k}\right|}{\left|z_{k}-x\right|}=0$, verifica-se que

$$
\lim _{k \rightarrow \infty} \frac{\left|h\left(z_{k}\right)-h\left(y_{k}\right)\right|}{\left|h\left(z_{k}\right)-h(x)\right|}=0
$$

\subsection{Vários tipos de homeomorfismos}

Estudamos aqui a relação existente entre as várias classes de homeomorfismos.

Lema 1.4.1 Se $h:[a, b] \rightarrow[c, d]$ é um difeomorfismo, entioo h é quase-simétrico.

\section{Prova:}

Se $h$ é um difeomorfismo então para todo $x$ em [a,b] verifica-se que

$$
\lim _{\epsilon \rightarrow 0} \frac{|h(x+\epsilon)-h(x)|}{\epsilon}=\lim _{\epsilon \rightarrow 0} \frac{|h(x)-h(x-\epsilon)|}{\epsilon}=h^{\prime}(x)
$$

e sendo $h^{\prime}(x) \neq 0$ tem-se que

$$
\lim _{\epsilon \rightarrow 0} \frac{|h(x+\epsilon)-h(x)|}{|h(x)-h(x-\epsilon)|}=1 .
$$

Entretanto se $h$ não é quase-simétrico, então existem duas seüências $\left\{x_{n}\right\}_{n=1}^{\infty}$ e $\left\{\epsilon_{n}\right\}_{n=1}^{\infty}$ tal que

$$
\frac{\left|h\left(x_{n}+\epsilon_{n}\right)-h\left(x_{n}\right)\right|}{\left|h\left(x_{n}\right)-h\left(x_{n}-\epsilon_{n}\right)\right|}>n .
$$


Pela compacidade do domínio de $h$ existe uma subseqüência convergente, à qual chamamos também $\left\{\epsilon_{n}\right\}_{n=1}^{\infty}$. Estamos interessados no caso em que $\epsilon_{n}$ tenda a zero. Seja $x$ o limite da correspondente subseqüência $\left\{x_{n}\right\}_{n=1}^{\infty}$, então verifica-se que

$$
\lim _{\epsilon \rightarrow 0} \frac{|h(x+\epsilon)-h(x)|}{|h(x)-h(x-\epsilon)|}=\infty .
$$

Isto contradiz o resultado anterior.

Lema 1.4.2 Se $h:[a, b] \rightarrow[c, d]$ é um homeomorfismo quase-simétrico então h é Hölder contínuo.

Prova: Seja $h:[a, b] \rightarrow[c, d]$ um homeomorfismo $h$-quase-simétrico. Então se $\alpha=\log _{2} \frac{1+K}{K}$ e $C=2^{2 \alpha}|h(b)-h(a)| /(b-a)^{\alpha}$, mostramos que

$$
|h(x)-h(y)| \leq C|x-y|^{\alpha}
$$

Suponhamos que $x<y$ e $y \geq \frac{a+b}{2}$. Se $y<\frac{a+b}{2}$, consideramos o homeomorfismo $g$ : $[a, b] \rightarrow[c, d]$ definido por $g(x)=h(a)+h(b)-h(a+b-x)$. Desta forma este caso se reduz ao caso anterior. Como na prova do Lema 1.3.1 definimos $\omega_{0}=a$ e indutivamente $\omega_{n+1}=\frac{\omega_{n}+y}{2}$. Então existe $N$ tal que $\omega_{N}<x \leq \omega_{N+1}$ e então resulta que

$$
\begin{aligned}
|h(x)-h(y)| & \leq\left(\frac{K}{1+K}\right)^{N}|h(a)-h(y)| \\
& \leq|h(b)-h(a)|\left(\frac{1}{2^{N}}\right)^{\log _{2} \frac{1+K}{K}} .
\end{aligned}
$$

Como

$$
\frac{|y-x|}{|y-a|} \geq \frac{1}{2^{N+1}}
$$

temos que

$$
|h(x)-h(y)| \leq|h(b)-h(a)| 2^{\alpha} \frac{|y-x|^{\alpha}}{|y-a|^{\alpha}} \leq \frac{|h(b)-h(a)| 2^{2 \alpha}}{(b-a)^{\alpha}}|y-x|^{\alpha} .
$$

Isto conclui a demonstração. 
Do que vimos até o momento concluímos, em particular, que no caso de um homeomorfismo $h:[a, b] \rightarrow[c, d]$ verificam-se que:

- Se $h$ é um difeomorfismo então $h$ é quase-simétrico.

- Se $h$ é um homeomorfismo quase-simétrico então $h$ é Hölder contínuo.

- Se $h$ é um homeomorfismo absolutamente contínuo então $h$ é diferenciável Lebesgue q.t.p.

\subsection{Exemplos}

Os exemplos abaixo mostram que as recíprocas dos Lemas da seção anterior não se verificam.

Exemplo 1.5.1 A função $h:[0,2] \rightarrow[0,2]$ definida por

$$
h(x)=\left\{\begin{array}{cc}
\sqrt{x} & \text { se } 0 \leq x \leq 1 \\
\sqrt{x-1}+1 & \text { se } 1 \leq x \leq 2
\end{array}\right.
$$

é Hölder contínua, mas não é quase-simétrica(veja a Figura 1.1).

Provamos que $h$ é $(\sqrt{3}, 1 / 2)$-Hölder contínua. Para isto consideramos três casos: Caso 1: Sejam $x, y$ em $[0,1] \operatorname{com} x<y$. Então

$$
|y-x|-|\sqrt{y}-\sqrt{x}|^{2}=-2 x+2 \sqrt{x y}>0
$$

e portanto

$$
|\sqrt{y}-\sqrt{x}|<|y-x|^{1 / 2} .
$$

Caso 2: Sejam $x, y$ em $[1,2] \operatorname{com} x<y$. Então $0<x-1<y-1<1$ e resulta que

$$
|h(y)-h(x)|=|\sqrt{y-1}-\sqrt{x-1}|<|y-x|^{1 / 2} .
$$




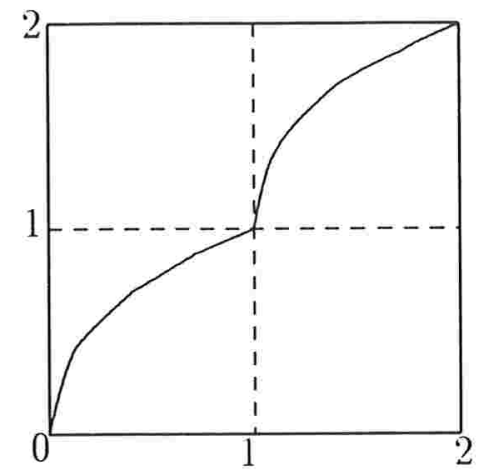

Figura 1.1:

Caso 3: Sejam $x$ em $[0,1)$ e $y$ em $(1,2]$. Como $h$ é um homeomorfismo temos que

$$
|h(y)-h(x)|=|h(y)-h(1)|+|h(1)-h(x)|<|y-1|^{1 / 2}+|1-x|^{1 / 2} .
$$

Mas como $|y-1|<|y-x|$ e $|x-1|<|y-x|$ temos que

$$
\begin{aligned}
\left(|y-1|^{1 / 2}+|1-x|^{1 / 2}\right)^{2} & =|y-1|+|1-x|+2|y-1|^{1 / 2}|1-x|^{1 / 2} \\
& <|y-x|+2|y-x|=3|y-x|
\end{aligned}
$$

e disto resulta que

$$
|h(y)-h(x)|<\sqrt{3}|y-x|^{1 / 2} .
$$

Desta forma concluímos que $h$ é $(\sqrt{3}, 1 / 2)$-Hölder contínua como queríamos. Entretanto, para $x=1$ temos que

$$
\frac{|h(1+\epsilon)-h(1)|}{|h(1)-h(1-\epsilon)|}=\frac{\sqrt{\epsilon}}{1-\sqrt{1-\epsilon}}
$$

e como este quociente tende para infinito quando $\epsilon$ tende a zero concluímos que $h$ não é quase-simétrica.

Exemplo 1.5.2 A função $h:[0,1 / 2] \rightarrow \mathbf{R}$ definida por

$$
h(x)=\left\{\begin{array}{cl}
\sqrt{\frac{1}{\log \left(\frac{1}{x}\right)}} & \text { se } x \in(0,1 / 2] \\
0 & \text { se } x=0
\end{array}\right.
$$


é absolutamente contínua mas não é Hölder contínua.

Sabemos que $h$ é diferenciável em $(0,1 / 2]$ e disto resulta que $h$ é absolutamente contínua em $[0,1 / 2]$. Provaremos que $h$ não é Hölder contínua. Para isto consideramos

$$
g_{1}(x)=\left\{\begin{array}{cc}
e^{-1 / x^{2}} & \text { se } x \neq 0 \\
0 & \text { se } x=0
\end{array}\right.
$$

e $g_{2}(x)=x^{\alpha} \operatorname{com} \alpha \geq 1$. Temos que $g_{1}(0)=g_{2}(0)=0 \mathrm{e}$

$$
g_{2}^{\prime}(x)-g_{1}^{\prime}(x)=\alpha x^{\alpha-1}+\frac{2 e^{-\frac{1}{x^{2}}}}{x^{3}}>0
$$

para todo $x>0$. Portanto $g_{2}(x)>g_{1}(x)$ para todo $x>0$. Como $h$ e $g_{1}$ são funções inversas uma da outra temos que $h(x)>x^{1 / \alpha}$ para todo $x>0$ e $\alpha \geq 1$. Então concluímos que $h$ não é Hölder contínua.

Exemplo 1.5.3 A função $h:[-1,1] \rightarrow[-1,1]$ definida por

$$
h(x)=\left\{\begin{array}{cl}
-x^{3}, & \text { se } x \in[-1,0] \\
x^{2}, & \text { se } x \in[0,1]
\end{array}\right.
$$

é um homeomorfismo de classe $C^{1}$ mas não é quase-simétrico. De fato, para $x=0$, temos que

$$
\frac{|h(x+\epsilon)-h(x)|}{|h(x)-h(x-\epsilon)|}=\frac{\epsilon^{2}}{\epsilon^{3}}
$$

e este quociente tende para infinito quando $\epsilon$ tende para zero.

Exemplo 1.5.4 Existem homeomorfismos $h:[0,1] \rightarrow[0,1]$ que são quase-simétricos mas não são deriváveis em um conjunto denso em $[0,1]$.

Um tal homeomorfismo $h:[0,1] \rightarrow[0,1]$ pode ser obtido como limite de uma seqüência de homeomorfismos quase-simétricos $h_{n}:[0,1] \rightarrow[0,1]$ a qual construímos indutivamente, a saber:

Passo 0. Definimos $h_{0}(x)=x$; para $x \in[0,1]$.

Passo $\mathbf{n}+\mathbf{1}$. Dado $h_{n}$ definimos $h_{n+1}$ como sendo o homeomorfismo que satisfaz as seguintes propriedades: (Veja a Figura 1.2) 
1. Para $1 \leq k \leq 2^{n+1}$. a restrição de $h_{n+1}$ a $\left[(k-1) 2^{-n-1}, k 2^{-n-1}\right]$ é uma função linear afim.

2. Para $1 \leq k \leq 2^{n}$ verifica-se que $h_{n+1}\left(2 k 2^{-n-1}\right)=h_{n}\left(k \cdot 2^{-n}\right)$.

3. Se $n$ é ímpar verifica-se que

$$
h_{n+1}\left((2 k-1) 2^{-n-1}\right)=\frac{1}{4} h_{n}\left((k-1) 2^{-n}\right)+\frac{3}{4} h_{n}\left(k 2^{-n}\right) .
$$

4. Se $n$ é par verifica-se que

$$
\begin{gathered}
h_{n+1}\left((2 k-1) 2^{-n-1}\right)=\frac{3}{4} h_{n}\left((k-1) 2^{-n}\right)+\frac{1}{4} h_{n}\left(k 2^{-n}\right) \\
\text { Caso n impar } \\
\hline k-1) 2^{-n}
\end{gathered}
$$
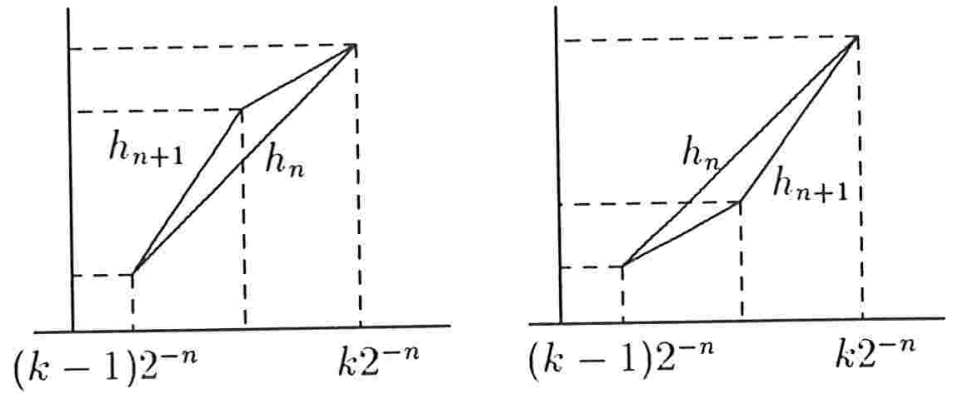

Figura 1.2:

Da definição acima resulta que para cada $n$, o homeomorfismo $h_{n+1}$ é definido dividindo cada intervalo de linearidade de $h_{n}$ em dois subintervalos de linearidade. A razão dos declives dos dois subintervalos gerados a partir de um intervalo de linearidade é 3 . E em geral, considerando apenas pontos de não-linearidade, verifica-se a seguinte propriedade: no caso $n$ par, para $1 \leq k<2^{n}$, temos que

$$
\begin{aligned}
& h_{n+1}\left(2(k-1) 2^{-n-1}\right)=h_{n}\left((k-1) 2^{-n}\right) \\
& h_{n+1}\left((2 k-1) 2^{-n-1}\right)=\frac{3}{4} h_{n}\left((k-1) 2^{-n}\right)+\frac{1}{4} h_{n}\left(k 2^{-n}\right)
\end{aligned}
$$




$$
\begin{aligned}
h_{n+1}\left(2 k 2^{-n-1}\right) & =h_{n}\left(k 2^{-n}\right) \\
h_{n+1}\left((2 k+1) 2^{-n-1}\right) & =\frac{3}{4} h_{n}\left(k 2^{-n}\right)+\frac{1}{4} h_{n}\left((k+1) 2^{-n}\right) \\
h_{n+1}\left(2(k+1) 2^{-n-1}\right) & =h_{n}\left((k+1) 2^{-n}\right)
\end{aligned}
$$

Deste fato resulta que se

$$
3\left|h_{n}\left(k 2^{-n}\right)-h_{n}\left((k-1) 2^{-n}\right)\right|=\left|h_{n}\left((k+1) 2^{-n}\right)-h_{n}\left(k 2^{-n}\right)\right|
$$

verifica-se que o quociente

$$
\frac{\left|h_{n+1}\left((i+1) 2^{-n-1}\right)-h_{n+1}\left(i 2^{-n-1}\right)\right|}{\left|h_{n+1}\left(i 2^{-n-1}\right)-h_{n+1}\left((i-1) 2^{-n-1}\right)\right|}
$$

é igual a 3 quando $i=2 k \pm 1$ e a 1 quando $i=2 k$. E se

$$
\left|h_{n}\left(k 2^{-n}\right)-h_{n}\left((k-1) 2^{-n}\right)\right|=3\left|h_{n}\left((k+1) 2^{-n}\right)-h_{n}\left(k 2^{-n}\right)\right|
$$

verifica-se que o quociente de (1.1) é 3 para $i=2 k \pm 1$ e para $i=2 k$ verifica-se que

$$
\frac{\left|h_{n+1}\left((2 k+1) 2^{-n-1}\right)-h_{n+1}\left(2 k 2^{-n-1}\right)\right|}{\left|h_{n+1}\left(2 k 2^{-n-1}\right)-h_{n+1}\left((2 k-1) 2^{-n-1}\right)\right|}=\frac{1}{9} \text {. }
$$

Neste caso, para $n+2$ temos que

$$
\begin{aligned}
h_{n+2}\left(2(2 k-1) 2^{-n-2}\right) & =h_{n+1}\left((2 k-1) 2^{-n-1}\right) \\
h_{n+2}\left((2(2 k-1)+1) 2^{-n-2}\right) & =\frac{1}{4} h_{n+1}\left((2 k-1) 2^{-n-1}\right)+\frac{3}{4} h_{n+1}\left(2 k 2^{-n-1}\right) \\
h_{n+2}\left(2(2 k) 2^{-n-2}\right) & =h_{n+1}\left(2 k 2^{-n-1}\right) \\
h_{n+2}\left((2(2 k)+1) 2^{-n-2}\right) & =\frac{1}{4} h_{n+1}\left(2 k 2^{-n-1}\right)+\frac{3}{4} h_{n+1}\left((2 k+1) 2^{-n-1}\right) \\
h_{n+2}\left(2(2 k+1) 2^{-n-2}\right) & =h_{n+1}\left((2 k+1) 2^{-n-1}\right)
\end{aligned}
$$

E resulta que

$$
\frac{\left|h_{n+2}\left((i+1) 2^{-n-2}\right)-h_{n+2}\left(i 2^{-n-2}\right)\right|}{\left|h_{n+2}\left(i 2^{-n-2}\right)-h_{n+2}\left((i-1) 2^{-n-2}\right)\right|}=\frac{1}{3}
$$

para $i=4 k \pm 1,4 k$. Para $n$ ímpar é semelhante. 
Disto resulta que, para cada $n$, a razão dos declives de cada dois intervalos de linearidade adjacentes é apenas 3 ou 9 , isto é, $h_{n}$ é 9-quase-simétrica. Segue a partir da definição que $h_{n}$ converge uniformemente para um homeomorfismo $h$ o qual também é 9quase-simétrico. Entretanto $h$ não é derivável no conjunto de todos os números racionais cujos denominadores são potências de 2 e este conjunto é denso em $[0,1]$.

Exemplo 1.5.5 Existem homeomorfismos $h:[0,1] \rightarrow[0,1]$ que sào quase-simétricos mas não são deriváveis em um conjunto de Cantor.

Para obter um tal homeomorfismo consideramos dois conjuntos de Cantor obtidos do seguinte modo: Sejam $0<\alpha<\beta<1$. Retiramos do intervalo $I=[0,1]$ o seu intervalo aberto médio $B_{1}^{0}$ tal que $\left|B_{1}^{0}\right|=\alpha|I|$. Às componentes conexas de $I \backslash B_{1}^{0}$ são denotadas por $L_{1}^{0}$ e $L_{2}^{0}$. Em seguida retiramos de cada $L_{j}^{0}$ o seu intervalo aberto médio $B_{j}^{1}$ tal que $\left|B_{j}^{1}\right|=\beta\left|L_{j}^{0}\right|$. Às componentes conexas de $\left(L_{1}^{0} \cup L_{2}^{0}\right) \backslash\left(B_{1}^{1} \cup B_{2}^{1}\right)$ são denotadas por $L_{1}^{1}, L_{2}^{1}, L_{3}^{1}$ e $L_{4}^{1}$. Continuamos, indutivamente, a retirar da partir do intervalo $L_{j}^{n}$, onde $j \in\left\{1, \cdots, 2^{n+1}\right\}$, o seu intervalo aberto médio $B_{j}^{n+1}$ tal que $\left|B_{j}^{n+1}\right|=\alpha\left|L_{j}^{n}\right|$ se $n$ é par e $\left|B_{j}^{n+1}\right|=\beta\left|L_{j}^{n}\right|$ se $n$ é impar. Às componentes conexas de $\left(\bigcup_{j=1}^{2 n+1} L_{j}^{n}\right) \backslash\left(\bigcup_{j=1}^{2 n+1} B_{j}^{n+1}\right)$ são denotadas por $L_{j}^{n+1} ; j=1, \cdots 2^{n+2}$. Então o conjunto

$$
K=I \backslash \bigcup_{n=0}^{\infty} \bigcup_{j=1}^{2^{n}} B_{j}^{n}
$$

é um conjunto de Cantor.

Repetindo este mesmo processo, desta vez retirando primeiro o intervalo aberto médio de proporção $\beta$ e em seguida o de proporção $\alpha$ e continuando de forma alternada como antes obtemos o conjunto

$$
\tilde{K}=[0,1] \backslash \bigcup_{n=0}^{\infty} \bigcup_{i=1}^{2^{n}} \tilde{B}_{i}^{n},
$$

onde $\tilde{B}_{i}^{n}$ é intervalo aberto médio retirado de $\tilde{L}_{j}^{n-1}$ na $n$-ésima etapa do processo. Chamamos $\tilde{L}_{j}^{n}$, onde $j \in\left\{1, \cdots, 2^{n+1}\right\}$, aos intervalos restantes da $n$-ésima etapa do pro-

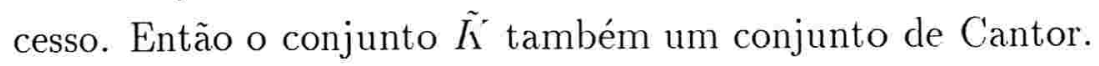


Da construçào de $K$ e $\tilde{K}$, temos a seguinte estimativa:

$$
\begin{array}{ll}
\left|B_{1}^{0}\right|=\alpha & \left|\tilde{B}_{1}^{0}\right|=\beta \\
\left|B_{j}^{1}\right|=\frac{(1-\alpha) \beta}{2} & \left|\tilde{B}_{j}^{1}\right|=\frac{(1-\beta) \alpha}{2} \\
\left|B_{j}^{2 m-1}\right|=\frac{(1-\alpha)^{m}(1-\beta)^{m-1} \beta}{2^{2 m-1}} & \left|\tilde{B}_{j}^{2 m-1}\right|=\frac{(1-\alpha)^{m-1}(1-\beta)^{m} \alpha}{2^{2 m-1}} \\
\left|B_{j}^{2 m}\right|=\frac{(1-\alpha)^{m}(1-\beta)^{m} \alpha}{2^{2 m}} & \left|\tilde{B}_{j}^{2 m}\right|=\frac{(1-\alpha)^{m}(1-\beta)^{m} \beta}{2^{2 m}} \\
\left|L_{j}^{2 m-1}\right|=\frac{(1-\alpha)^{m}(1-\beta)^{m-1}}{2^{2 m-1}} & \left|\tilde{L}_{j}^{2 m-1}\right|=\frac{(1-\alpha)^{m-1}(1-\beta)^{m}}{2^{2 m-1}} \\
\left|L_{j}^{2 m}\right|=\frac{(1-\alpha)^{m}(1-\beta)^{m}}{2^{2 m}} & \left|\tilde{L}_{j}^{2 m}\right|=\frac{(1-\alpha)^{m}(1-\beta)^{m}}{2^{2 m}}
\end{array}
$$

Agora, podemos definir um homeomorfismo quase-simétrico $h:[0,1] \rightarrow[0,1]$ tal que $h\left(K^{\prime}\right)=\tilde{K}$. Procedemos do seguinte modo: a restrição $\left.h\right|_{\bar{B}_{j}^{n}}$ é uma função linear afim crescente que aplica $\bar{B}_{j}^{n}$ bijetivamente em $\overline{\tilde{B}_{j}^{n}}$, para todo $j=1, \cdots, 2^{n}$ e $n \geq 0$. Em seguida estendemos $h$ a $[0,1]$ por continuidade. Então temos que em cada intervalo $\bar{B}_{j}^{n}$ a função $h$ tem declive $\frac{(1-\beta) \alpha}{(1-\alpha) \beta}$ quando $n$ é ímpar e $\frac{\beta}{\alpha}$ quando $n$ é par. Como vemos na estimativa acima o quociente $\frac{\left|\tilde{L}_{j}^{n}\right|}{\left|L_{j}^{n}\right|}$ é $\frac{(1-\beta)}{(1-\alpha)}$ no caso $n$ ímpar e 1 no caso $n$ par. Ou seja em $[0,1]$ a função $h$ possui seu declive no máximo $\frac{\beta}{\alpha}$ e no mínimo $\frac{(1-\beta) \alpha}{(1-\alpha) \beta}$ Portanto, disto resulta que $h$ estendida é um homeomorfismo $\frac{(1-\alpha) \beta^{2}}{(1-\beta) \alpha^{2}}$-quase-simétrico. Por outro lado é fácil ver que $h$ não é derivável em $K$.

Em geral, dadas duas seqüências $\left\{\alpha_{n}\right\}_{n=1}^{\infty}$ e $\left\{\tilde{\alpha}_{n}\right\}_{n=1}^{\infty} \operatorname{com} 0<\alpha_{n}, \tilde{\alpha}_{n}<1$ para todo $n$, sejam $K_{\alpha}$ e $K_{\tilde{\alpha}}$ conjuntos de Cantor obtidos, retirando os intervalos abertos médios de proporção $\alpha_{n}, \tilde{\alpha}_{n}$ respectivamente na $n$-ésima etapa do processo. Então a função definida como no exemplo anterior conjuga $K_{\alpha}$ e $K_{\dot{\alpha}}$ quase-simetricamente, se existir $M>1$ tal que para todo $n$ verifica-se que

$$
\frac{1}{M} \leq \frac{\alpha_{n}}{\tilde{\alpha}_{n}} \Pi_{i=1}^{n-1}\left(\frac{1-\alpha_{i}}{1-\tilde{\alpha}_{i}}\right) \leq M .
$$

No entanto esta conjugação não é derivável em $K_{\alpha}$.

Existem também muitos exemplos de conjugações entre duas funções do círculo ou do intervalo que são quase-simétricas mas não são absolutamente contínuas. Dentre estas 
conjugações existem vários exemplos interessantes de homeomorfismos, a seguir citaremos alguns. Inicialmente observamos que se $g: S^{1} \rightarrow S^{1}$ é um difeomorfismo $C^{2}$ cujo número de rotação $\rho_{g}$ é irracional existe uma conjugação $h: S^{1} \rightarrow S^{1}$ entre $f$ e $\mathcal{R}_{\rho_{g}}$, onde $\mathcal{R}_{\rho_{g}}: S^{1} \rightarrow S^{1}$ é a rotaçào de ângulo $\rho_{g}$. No caso em que $g$ é de classe $C^{3}$ e $\rho_{g}$ é Diophantino esta conjugaçào é de classe $C^{1}$, veja [YJ]. No caso em que $g_{1}, g_{2}: S^{1} \rightarrow S^{1}$ são funções de classe $C^{1+\alpha}$ expansivas e do mesmo grau $d \geq 2$, elas são quase-simetricamente conjugadas, veja $[\mathrm{SM}]$ e $[\mathrm{MS}]$. Por outro lado se $g_{1}, g_{2}$ são conjugadas por um homeomorfismo absolutamente contínuo com inversa absolutamente contínua resulta que $g_{1}, g_{2}$ sào $C^{1}$ conjugadas, veja [SS]. Portanto $D h\left(g_{1}^{n}(x)\right) D g_{1}^{n}(x)=D g_{2}^{n}(h(x)) D h(x)$ para todo $x \in S^{1}$ e conseqüentemente os autovalores dos pontos periódicos correspondentes de $g_{1}$ e $g_{2}$ coincidem. Então se $g_{1}, g_{2}$ de classe $C^{1+\alpha}$ possuem pontos periódicos correspondentes com autovalores diferentes elas são quase-simetricamente conjugadas. No entanto esta conjugação não é derivável na órbita negativa destes pontos periódicos não sendo portanto absolutamente contínua.

Em [BA] foi provado que existe uma função quase-simétrica que é puramente singular, isto é, tem derivada nula em um conjunto com medida de Lebesgue total. Neste caso a referida função não é absolutamente contínua. De fato, se ela é absolutamente contínua então, do Teorema 1.1.3. é uma função constante que não é um homeomorfismo. Deste fato resulta que a quase-simetria não implica a continuidade absoluta. Observamos que no caso de homeomorfismos diferenciáveis, Hölder contínuos, absolutamente contínuos o homeomorfismo inverso pode não possuir estas propriedades. No entanto no caso de homeomorfismos quase-simétricos o homeomorfismo inverso também é quase-simétrico. Veja em [MS] outros comentários sobre conjugação quase-simétrica ou absolutamente contínua em Dinâmica unidimensional. 


\section{Capítulo 2}

\section{Dinâmica de Funções Unimodais}

Neste capítulo estudamos alguns aspectos da dinâmica unidimensional. Em particular exibimos uma propriedade importante satisfeita pelas funções unimodais de classe $C^{2}$ que são quase-simetricamente conjugadas a funções tendas.

\subsection{Conceitos básicos}

O sistema dinâmico gerado por uma função unimodal $f: I \rightarrow I$ envolve infinitas funções, as iteradas de $f$, definidas indutivamente por $f^{1}=f$ e $f^{n+1}=f \circ f^{n}$. Paralelamente dado um ponto $x$ em $[-1,1]$, temos as noções de órbita postiva de $x$

$$
\operatorname{orb}_{f}^{+}(x):=\left\{y \in I ; y=f^{n}(x), n \geq 0\right\}
$$

órbita negativa de $x$

$$
\operatorname{orb}_{f}^{-}(x):=\left\{y \in I ; f^{n}(y)=x, n \geq 0\right\}
$$

e grande órbita de $x$

$$
\operatorname{orb}_{f}(x):=\left\{y \in I ; f^{n}(y)=f^{m}(x), n, m \geq 0\right\}
$$


Uma das metas principais da teoria dos sistemas dinâmicos é descrever as propriedades assintóticas das órbitas de um dado sistema. Fixemos uma função unimodal $f: I \rightarrow I$ e definimos o conjunto $\omega$-limite de um ponto $x \in I$ (o qual denotamos por $\left.\omega_{f}(x)\right)$ como sendo o conjunto dos pontos de acumulaçào da seqüência $\left\{f^{n}(x)\right\}_{n=0}^{\infty}$. Dito de outra forma temos que

$$
\omega(x)=\bigcap_{k=1}^{\infty} \overline{\bigcup_{n=k}^{\infty} f^{n}(x)} .
$$

No caso de uma função unimodal o comportamento da órbita do ponto crítico $c$ determina vários aspectos da dinâmica gerada por esta funçào. Entâo a estrutura do $\omega$-limite do ponto crítico $c$, o conjunto $\omega_{f}(c)$, será de particular interesse.

Definição 2.1.1 Um ponto $p \in I$ é chamado um ponto periódico de período $n$ de uma funçâo unimodal $f$ se $f^{i}(p) \neq p$ para $0<i<n$ e $f^{n}(p)=p$. A bacia de atração de um ponto periódico $p$, a qual denotaremos por $B_{f}(p)$, é definida por:

$$
B_{f}(p):=\left\{y: \omega(y)=\operatorname{orb}_{f}^{+}(p)\right\} .
$$

A bacia imediata de atração de $p$ é a componente conexa de $B_{f}(p)$ que contém $p$. Um ponto periódico $p$ é chamado atrator se a sua bacia de atração contém um intervalo aberto.

Um intervalo $J \subset I$ é dito intervalo errante para $f$ se $J$ não está contido em uma bacia de atrator periódico e os intervalos $f^{n}(J), n \geq 0$ têm interior dois a dois disjuntos.

Lema 2.1.1 (Princípio da Contração) Seja $J$ um intervalo tal que $\inf _{n \geq 0}\left|f^{n}(J)\right|=0$. Então, ou J é um intervalo errante ou está contido na bacia de atração de algum atrator periódico.

Prova: Se $T=\cup_{n \geq 0} f^{n}(J)$ então $f^{k}(T) \subset T$ para todo $k \geq 0$. Consideramos dois casos:

Caso 1: Suponhamos que existam $n>0$ e uma componente $U$ de $T$ tais que $f^{n}(U) \cap$ $U \neq \phi$. Como $T$ é invariante temos que $f^{n}(U) \subset U$ e portanto $f^{n}$ possui um ponto fixo 
em $\bar{U}$. Se $f^{n}$ possui um ponto fixo $p$ no interior de $U$ entào existe $i$ tal que $p \in \overline{f^{i}(J)}$. Como $\inf \left\{\left|f^{n}(J)\right| ; n \geq 0\right\}=0$ resulta que $J$ está contido na bacia de atração de $p$. Se $f^{n}$ não possui um ponto fixo no interior de $U$ então $f^{n}$ possui um ponto fixo $p$ no bordo de $U$ e neste caso podemos ter que $f^{n}(U) \subsetneq U$, ou que $f^{n}(U)=U$. Se $f^{n}(U) \subsetneq U$ resulta que $J$ está na bacia de atração de $p$ e se $f^{n}(U)=U$ resulta que $f^{n}$ possui um outro ponto fixo $q$ no bordo de $U$. Então $J$ está contido na bacia de atração de $p$ ou de $q$.

Caso 2: Suponhamos que para toda componente $U$ de $T, f^{n}(U) \cap U=\phi$, para todo $n \geq 1$. Então como $T$ é invariante $f^{n}(U) \cap f^{m}(U)=\phi$, para todo $n>m \geq 0$. Isto implica que, ou $U$ é intervalo errante ou $U$ converge para uma órbita periódica atratora.

Do Princípio de Contração temos um corolário imediato.

Corolário 2.1.1 Se uma funçâo unimodal $f: I \rightarrow I$ não admite intervalos errantes então para todo $\epsilon>0$ existe $\delta>0$ tal que $\left|f^{n}(J)\right|>\delta$ para qualquer $n \geq 0$ e $|J|>\epsilon$.

Definição 2.1.2 Um intervalo $J \subset I$ é chamado intervalo periódico de $f$ se:

1. Existe $n \geq 1$ tal que $f^{n}(J) \subset J$ e $f^{n}(\partial J) \subset \partial J$.

2. Os intervalos $J, \cdots, f^{n-1}(J)$ possuem interior dois a dois disjuntos.

O inteiro $n \geq 1$ é chamado período de $J$.

Observamos que se $J$ é um intervalo $n$-periódico da função unimodal $f$ e $\bigcup_{i=0}^{n-1} f^{i}(J)$ contém o ponto crítico $c$, então $\left.f^{n}\right|_{J}: J \rightarrow J$ é também uma função unimodal. Neste caso dizemos que $f$ é renormalizável e que $\left.f^{n}\right|_{J}$ é uma renormalização de $f$. No caso em que $f$ possui um número infinito de intervalos periódicos chamamos-na infinitamente renormalizável e no caso contrário chamamos-na finitamente renormalizável. No caso em que $f$ não possui intervalos periódicos chamamos-na não-renormalizável. 
Uma função tenda $T_{a} \operatorname{com} \sqrt{2}<a \leq 2$ é nào-renormalizável e se $\sqrt[2^{m+1}]{2}<a \leq$ $\sqrt[2 m]{2}$ para algum $m \geq 1$ então $T_{a}$ é finitamente renormalizável e possui intervalos $2^{i}$ periódicos para $i=1, \cdots, m$. Disto resulta em particular que não existem funções tendas infinitamente renormalizáveis.

Um exemplo de uma função unimodal infinitamente renormalizável é a função de Feigenbaum-Tresser e um outro exemplo de função unimodal não-renormalizável é a função de Fibonacci, veja seção 3.3

\subsection{Conjugação quase-simétrica}

A relação de equivalência natural para expressarmos a noção de "mesma dinâmica" é o que chamamos conjugação, a saber: duas funções unimodais $f$ e $g$ dos intervalos $I_{f}$ e $I_{g}$ respectivamente, são conjugadas se existe um homeomorfismo $h: I_{f} \rightarrow I_{g}$ tal que $h \circ f=g \circ h$. Um tal homeomorfismo, chamado uma conjugação entre $f$ e $g$, aplica órbitas de $f$ em órbitas de $g$ e preserva as suas propriedades topológicas. No entanto muitas vezes temos interesse em propriedades métricas e geométricas do sistema. Então quanto mais regularidade possui a conjugação $h$ mais propriedades serão preservadas, por exemplo: $h$ sendo absolutamente contínua temos que $f$ possui uma medida invariante absolutamente contínua com respeito à medida de Lebesgue então o mesmo se verifica para $g$. No caso em que $h$ é quase-simétrica Sands e Nowicki provaram em [SN] que $f$ satisfaz a condição de Collet-Eckmann se, e somente se, o mesmo ocorre para $g$.

Dada uma função unimodal finitamente renormalizável podemos renormalizá-la um número finito de vezes e obter uma função unimodal não-renormalizável. Nos restringimos então ao caso de funções unimodais que são não-renormalizáveis. Assumimos também que $f$ não possui atrator periódico e não possui intervalos errantes. Nestas condições sabemos que $f$ é topologicamente conjugada a uma função tenda $T_{a}$ com $\sqrt{2}<a \leq 2$. Com bastante freqüência esta conjugação é Hölder contínua. De fato, 
de $[N P]$ resulta que existe um conjunto de parâmetros em $(\sqrt{2}, 2]$, digamos $\mathcal{P}$, tal que $\lambda(\mathcal{P})>0$ e para todo $a \in \mathcal{P}$ tem-se que a função quadrática $q_{a}$ é Hölder continuamente conjugada a uma função tenda $T_{a}$. Infelizmente nào podemos esperar que estas conjugações sejam muito mais regulares do que Hölder continuas, veremos que para um conjunto de parâmetros em $(\sqrt{2}, 2]$, digamos $\mathcal{Q}$, tal que $\lambda(\mathcal{Q})=1$ e para todo $a \in \mathcal{Q}$ tem-se que $T_{a}$ nunca é quase-simetricamente conjugada a uma função unimodal de classe $C^{2}$. O Lema a seguir estabelece uma primeira propriedade a qual as funções unimodais de classe $C^{2}$ que são quase-simetricamente conjugadas a funções tendas devem satisfazer.

Lema 2.2.1 Seja $h: I \rightarrow I_{a}$ uma conjugação entre uma função unimodal $f \in$ uma função tenda $T_{a}\left(h \circ f=T_{a} \circ h\right)$ com $\sqrt{2}<a \leq 2$. Se $h \in h^{-1}$ são $(a, C)$-Hölder contínuas então existe $M$ tal que para todo intervalo $J \subset I=[-1,1]$ verifica-se que

$$
L(J)=\sum_{i=0}^{\tau(J)}\left|f^{i}(J)\right| \leq M
$$

onde $\tau(J)=\max \left\{i ;\left.f^{i}\right|_{J}\right.$ é monótona $\}$.

Prova: Tomamos $M$ tal que $\frac{1}{m^{2}}>C 2^{\alpha(1-m / 2)}$ para todo $m \geq M-2$ e suponhamos por contradição que existe $J$ tal que $L(J)>M$. Então resulta que

$$
M<L(J)=\sum_{i=0}^{\tau(J)}\left|f^{i}(J)\right| \leq \tau(J) .
$$

Afirmamos que existe $i$ tal que

$$
\tau(J)-i \geq M-2 \text { e }\left|f^{i}(J)\right|>\frac{1}{(\tau(J)-i)^{2}} .
$$

De fato, se não for este o caso verifica-se que

$$
L(J) \leq M-2+\sum_{i=0}^{\tau(J)-M+2} \frac{1}{(\tau(J)-i)^{2}}<M
$$


Da definição de $\tau(J)$ sabemos que $T_{a}$ expande o comprimento de $h(J)$ com o fator $a>\sqrt{2}$ até $\tau(J)$ iterações. Então escrevendo $m=\tau(J)-i$ temos que

$$
\left|h\left(f^{i}(J)\right)\right|=\left|h\left(f^{\tau(J)-m}(J)\right)\right| \leq a^{-m}\left|h\left(f^{\tau(J)}(J)\right)\right| \leq 2 \sqrt{2}^{-m} .
$$

Assumimos que $c=h(c)=0$ e que $f^{i}(J)=(x, y) \subset[0,1]$. Entào $h((x, y)) \subset[0,1]$ e pela escolha de $M$,

$$
\begin{aligned}
|x-y| & \geq \frac{1}{m^{2}}>C 2^{\alpha(1-m / 2)} \\
& \geq C \cdot\left|h\left(f^{i}(J)\right)\right|^{\alpha}=C|h(x)-h(y)|^{\alpha}
\end{aligned}
$$

Portanto $h^{-1}$ não pode ser Hölder contínua. Isto contradiz o fato de que $h^{-1}$ é $(\alpha, C)$-Hölder contínua.

Dada uma função unimodal $f$ dizemos que seu ponto crítico $c$ tem ordem $l$ se existir um difeomorfismo $\psi$, de classe $C^{\infty}$ tal que $\psi(0)=0$ e $f(x)=f(c)-|\psi(x-c)|^{l}$. No caso em que a função unimodal é de classe $C^{2}$ tem-se que $l \geq 2$. O ponto crítico de uma função unimodal será dito não-flat no caso em que a sua ordem for finita.

Lema 2.2.2 Seja $f: I \rightarrow I$ uma função unimodal de classe $C^{2}$ cujo ponto crítico c tem ordem $l \geq 2$. Entâo existem $1<l_{0}<l$ e uma vizinhança $V \ni$ c tais que para todo $x \in V$ e todo $y \in(c, x)$ se $f(x)<f(y)<\frac{f(c)+f(x)}{2}$ tem-se que

$$
\frac{|x-y|}{|x-c|} \leq \frac{1}{l_{o}} \frac{|f(x)-f(y)|}{|f(x)-f(c)|} .
$$

Prova: Assumimos $c=f(c)=0$ e consideramos $f(x)=|\psi(x)|^{l}$. Tomamos $V$ uma vizinha de $c$ tal que para todo $x \in V$ verifica-se que $f^{\prime \prime}(x)>0$ então temos que $\psi^{\prime \prime}(x)>0$ ou $\psi^{\prime \prime}(x)<0$ para todo $x \in V$. Pelo teorema do valor médio:

$$
|f(x)-f(y)|=\left|x-y\left\|f^{\prime}(\xi)|=l| x-y\right\| \psi^{\prime}(\xi)\right||\psi(\xi)|^{l-1}
$$


onde $0 \leq|y| \leq|\xi| \leq|x|$. Como $f(y) \geq f(x) / 2$ resulta que $|\psi \cdot(\xi)| \geq|\zeta \cdot(y)| \geq$ $|\psi(x)|(1 / 2)^{1 / l}$ e então concluimos que

$$
|f(x)-f(y)| \geq l|x-y|\left(\frac{1}{2}\right)^{(l-1) / l}\left|\psi^{\prime}(\xi)\right||\psi(x)|^{l-1} .
$$

Da escolha de $V$ temos que $\left|\psi^{\prime}(\xi)\right|>\left|\psi^{\prime}(x)\right| /|x|$ e portanto

$$
\frac{|f(x)-f(y)|}{|f(x)-f(c)|} \geq l \frac{|x-y|}{|x-c|}\left(\frac{1}{2}\right)^{(l-1) / l}
$$

Tomando $l_{o}(l)=l\left(\frac{1}{2}\right)^{(l-1) / l}$ temos que $\frac{d l_{o}(l)}{d l}=\left(\frac{1}{2}\right)^{(l-1) / l}\left(1-\frac{\log 2}{l}\right)$ e portanto $l_{o}(l)$ é uma função crescente para $l>\log 2$. Como $l_{0}(2)=\sqrt{2}$ concluimos que $l_{0}(l) \geq \sqrt{2}$ para todo $l \geq 2$. 


\section{Capítulo 3}

\section{Principais Instrumentos}

No estudo de propriedades métricas do sistema dinâmico gerado pelas iterações de uma função unimodal $f$ é indispensável que se tenha estimativas da distorção ou da nãolinearidade das iteradas $f^{n}$ em certos intervalos. Neste capítulo introduzimos ferramentas importantes que nos permitem ter estas estimativas.

\subsection{Razão cruzada}

Definição 3.1.1 Sejam $J, T$ dois intervalos tais que $J \subset T \in T \backslash J$ possui duas componentes conexas $L$ e R. Dizemos então que $T$ é uma $\delta$-vizinhança de $J$ se $|L| \geq \delta|J| e$ $|R| \geq \delta|J|$.

Definição 3.1.2 Seja $g:[a, b] \rightarrow[c, d]$ uma função de classe $C^{1}$. Se g não possui pontos críticos, definimos a distorção de $g$ em um intervalo $U \subset[a, b]$, denotada por dist $(g, U)$, do seguinte modo:

$$
\operatorname{dist}(g, U):=\sup \left\{\frac{\left|g^{\prime}(x)\right|}{\left|g^{\prime}(y)\right|} ; x, y \in U^{\prime}\right\}
$$


A quantidade $\operatorname{dist}(g, U)$ é uma medida de quanto a função $g$ distorce a razão de dois intervalos adjacentes $J_{1}, J_{2}$ contidos em $U$. De fato definimos a raz̃o de $J_{1}, J_{2}$ por:

$$
R\left(J_{1}, J_{2}\right)=\frac{\left|J_{1}\right|}{\left|J_{2}\right|}
$$

Consideramos então o seguinte quociente

$$
S\left(g, J_{1}, J_{2}\right)=\frac{R\left(g\left(J_{1}\right), g\left(J_{2}\right)\right)}{R\left(J_{1}, J_{2}\right)} .
$$

Verifica-se então, no caso em que $g$ é de classe $C^{1}$, que se dist $(g . U) \leq \Lambda^{\prime}$ resulta que $S\left(g, J_{1}, J_{2}\right) \leq K$ para quaisquer intervalos adjacentes $J_{1}, J_{2}$. A recíproca deste fato também é verdadeira. O caso em que estamos mais interessados é o caso em que $g$ é certas iteradas de uma função unimodal. Então a presença de pontos críticos dificulta obtermos boas estimativas para $\operatorname{dist}(g, U)$ e neste caso tanto $\operatorname{dist}(g, U) \operatorname{como} S\left(g, J_{1}, J_{2}\right)$ podem ser arbitrariamente grandes. Introduzimos então a razão cruzada de três intervalos adjacentes.

Definição 3.1.3 Sejam $J \subset T$ intervalos limitados tais que $T \backslash J$ possui duas componentes conexas $L$ e R. Então definimos a razão cruzada desses intervalos por

$$
D(T, J)=\frac{|J||T|}{|L||R|}
$$

Se $g: T \rightarrow N$ é uma função contínua e monótona, definimos

$$
B(g, T, J)=\frac{D(g(T), g(J))}{D(T, J)} .
$$

Observamos que se $J=[x, y] \subset T$ então

$$
d_{T}(x, y):=\log (1+D(T, J))=\log \left(1+\frac{|J|}{|L|}\right)+\log \left(1+\frac{|J|}{|R|}\right)
$$

define uma métrica no intervalo $T$ a qual é chamada de métrica de Poincaré do intervalo $T$. 
Seja $\phi: \mathbf{R} \rightarrow \mathbf{R}$ uma transformação de Möbius, isto é, uma função definida por

$$
\phi(x)=\frac{a x+b}{c x+d}
$$

onde $a, b, c, d$ são números reais tais que $a d-b c \neq 0$. Verifica-se então que $\phi$ preserva a razão cruzada de três intervalos, ou seja, $B(\phi, T, J)=1$. Também verifica-se que

$$
d_{\phi(T)}(\phi(x), \phi(y))=d_{T}(x, y)
$$

o que significa que $\phi$ é uma isometria entre os intervalos $T$ e $\phi(T)$ munidos das suas respectivas métricas de Poincaré.

A seguir introduzimos a derivada de Schwarz que nos permiti definir uma importante classe de funções $g: T \rightarrow g(T)$ para as quais $B(g, T, J) \geq 1$. Estas funções são expansões se consideramos $T$ e $g(T)$ munidos das suas respectivas métricas de Poincaré.

Definição 3.1.4 Se $g:[a, b] \rightarrow[c, d]$ é uma função de classe $C^{3}$ definimos a sua derivada de Schwarz por

$$
S(g)(x)=\frac{g^{\prime \prime \prime}(x)}{g^{\prime}(x)}-\frac{3}{2}\left(\frac{g^{\prime \prime}(x)}{g^{\prime}(x)}\right)^{2},
$$

se $g^{\prime}(x) \neq 0$. Se $g^{\prime}(x)=0$ definimos $S g(x)=-\infty$. Dizemos que $g$ tem derivada de Schwarz negativa se $S g(x)<0$ para todo $x$. Uma funçâo unimodal com derivada de Schwarz negativa é chamada S-unimodal.

Segue-se facilmente que se $\phi$ é uma transformação de Möbius então $S \phi=0$. Se $P(x)$ é um polinômio com coeficientes reais cujas raízes são todas reais e distintas então verifica-se que $S P<0$. A classe das funções $S$-unimodais forma um conjunto aberto na topologia $C^{3}$.

Lema 3.1.1 Sejam $f:[a, b] \rightarrow \mathbf{R}$ e $g:[c, d] \rightarrow \mathbf{R}$ funçôes de classe $C^{3}$ tais que $g([c, d]) \subset[a, b]$ então

$$
S(f \circ g)(x)=(S f \circ g(x)) \cdot\left(g^{\prime}(x)\right)^{2}+S g(x)
$$


Em particular se $S f<0$ e $S g<0$ resulta que $S(f \circ g)<0$.

Prova: Usando a regra da cadeia, verifica-se que

$$
\begin{gathered}
(f \circ g)^{\prime}(x)=f^{\prime}(g(x)) g^{\prime}(x) \\
(f \circ g)^{\prime \prime}(x)=f^{\prime \prime}(g(x))\left(g^{\prime}(x)\right)^{2}+f^{\prime}(g(x)) g^{\prime \prime}(x) \\
(f \circ g)^{\prime \prime \prime}(x)=f^{\prime \prime \prime}(g(x))\left(g^{\prime}(x)\right)^{3}+3 f^{\prime \prime}(g(x)) g^{\prime}(x) g^{\prime \prime}(x)+f^{\prime}(g(x)) g^{\prime \prime \prime}(x) .
\end{gathered}
$$

Portanto temos que

$$
\begin{aligned}
S(f \circ g)(x) & =\frac{f^{\prime \prime \prime}(g(x))\left(g^{\prime}(x)\right)^{2}}{f^{\prime}(g(x))}+3 \frac{f^{\prime \prime}(g(x)) g^{\prime \prime}(x)}{f^{\prime}(g(x))}+\frac{g^{\prime \prime \prime}(x)}{g^{\prime}(x)} \\
& -\frac{3}{2}\left(\frac{f^{\prime \prime}(g(x)) g^{\prime}(x)}{f^{\prime}(g(x))}+\frac{g^{\prime \prime \prime}(x)}{g^{\prime}(x)}\right)^{2} \\
& =\left[\frac{f^{\prime \prime \prime}(g(x))}{f^{\prime}(g(x))}-\frac{3}{2}\left(\frac{f^{\prime \prime}(g(x))}{f^{\prime}(g(x))}\right)^{2}\right]\left(g^{\prime}(x)\right)^{2}+\frac{g^{\prime \prime \prime}(x)}{g^{\prime}(x)}-\frac{3}{2}\left(\frac{g^{\prime \prime}(x)}{g^{\prime}(x)}\right)^{2} \\
& =S f(g(x))\left(g^{\prime}(x)\right)^{2}+S g(x) .
\end{aligned}
$$

De acordo com o Lema acima temos que a composição de duas funções com derivada de Schwarz negativa resulta em uma função com derivada de Schwarz negativa. Este fato faz da classe das funções com derivada de Schwarz negativa uma classe de particular interesse para sistemas dinâmicos.

Lema 3.1.2 Se $S g<0$, entâo $\left|g^{\prime}(x)\right|$ nào possui valor mínimo local positivo.

Prova: Suponhamos que $x_{0}$ seja ponto crítico de $g^{\prime}(x)$, isto é $g^{\prime \prime}\left(x_{0}\right)=0$. Como $S g\left(x_{0}\right)<0$, temos que $g^{\prime \prime \prime}\left(x_{0}\right) / g^{\prime}\left(x_{0}\right)<0$. Isto prova que $g^{\prime \prime \prime}\left(x_{0}\right)$ e $g^{\prime}\left(x_{0}\right)$ têm sinais opostos.

Lema 3.1.3 Se $g:[a, b] \rightarrow R$ é uma função S-unimodal e monótona então para todos intervalos $J \subset T \subset[a, b]$ temos que $B(g . T, J)>1$. 
Prova: Sejam $T=\left[x_{0}, x_{1}\right], J=\left[y_{0}, y_{1}\right]$ e $\phi$ uma transformação de Möbius tal que $\phi \circ g$ fixa as extremidade de $T$ e $y_{0}$. Disto resulta que $\phi\left(g\left(y_{1}\right)\right)>y_{1}$. De fato, suponhamos por absurdo que $\phi\left(g\left(y_{1}\right)\right) \leq y_{1}$. Então, pelo Teorema do valor médio, existem pontos $t_{0} \in\left[x_{0}, y_{0}\right], t_{1} \in\left[y_{0}, y_{1}\right]$ e $t_{2} \in\left[y_{1}, x_{1}\right]$ tais que

$$
\begin{gathered}
D(\phi \circ g)\left(t_{0}\right)=\frac{\phi\left(g\left(y_{0}\right)\right)-\phi\left(g\left(x_{0}\right)\right)}{y_{0}-x_{0}}=1, \\
D(\phi \circ g)\left(t_{1}\right)=\frac{\phi\left(g\left(y_{1}\right)\right)-\phi\left(g\left(y_{0}\right)\right)}{y_{1}-y_{0}} \leq 1
\end{gathered}
$$

$\mathrm{e}$

$$
D(\phi \circ g)\left(t_{2}\right)=\frac{\phi\left(g\left(x_{1}\right)\right)-\phi\left(g\left(y_{1}\right)\right)}{x_{1}-y_{1}} \geq 1 .
$$

Isto contradiz o Lema 3.1.2. Portanto $\phi\left(g\left(y_{1}\right)\right)>y_{1}$ e deste fato conjuntamente com a definição de $\phi$ concluímos que,

$$
\frac{|\phi(g(T))|}{|T|}=\frac{|\phi(g(L))|}{|L|}=1
$$

e

$$
\frac{|\phi(g(J))|}{|J|}>\frac{|\phi(g(R))|}{|R|} .
$$

Portanto,

$$
B(\phi \circ g, T, J)=\frac{\frac{|\phi(g(T) \mid)|}{|T|} \frac{|\phi(g(J))|}{|J|}}{\frac{|\phi(g(L))| \mid}{|L|(g(R)) \mid}}=\frac{\frac{|\phi(g(J))|}{|R|}}{|| J \mid} \mid \frac{|\phi(g(R))|}{|R|}>1 .
$$

Também temos que

$$
B(\phi \circ g, T, J)=B(\phi, g(T), g(J)) \cdot B(g, T, J)
$$

e como $\phi$ preserva a razão cruzada resulta que $B(g, T, J)>1$. 


\subsection{Princípios de Köbe}

Agora retornamos ao problema de estimar quanto uma função pode distorcer a razão cruzada de três intervalos. A razão cruzada de três intervalos desempenha um importante papel neste contexto e permite-nos obter os Princípios de Köbe abaixo os quais constituem ferramentas de extrema importância para o estudo de propriedades métricas dos sistemas dinâmicos unidimensionais.

Teorema 3.2.1 (Princípios de Köbe) Seja $g:[a, b] \rightarrow[c, d]$ um difeomorfismo de classe $C^{1}$ tal que para todos os intervalos $T=(x, y) \in J=(v, w)$ com $J \subset T \subset[a, b]$ tem-se que $B(g, T, J) \geq c_{0}>0$. Entâo verifica-se que

1. (Princípio da raiz quadrada) - Verifica-se que

$$
\frac{|g(T)|}{|T|} \geq \sqrt{c_{0} g^{\prime}(x) g^{\prime}(y)} .
$$

2. (Princípio do mínimo) - Existe constante $c_{1}$ que depende apenas de $c_{0}$ tal que para todo $\alpha \in T$ verifica-se que

$$
\left|g^{\prime}(\alpha)\right| \geq c_{1} \min \left\{\left|g^{\prime}(\beta)\right| ; \beta \in \partial T\right\} .
$$

3. (Princípio macroscópico) - Dado $\delta>0$ existe $r>0 \in M<\infty$ dependendo apenas de $\delta$ e $c_{0}$ tais que, se $g(T)$ é uma $\delta$-vizinhança de $g(J)$ então $T$ é uma $r$-vizinhança de $J$ e dist $(g, J) \leq M$.

4. (Princípio de Köbe unilateral) - Dado $\delta>0$ existe $c_{2}$ que depende apenas de $\delta$ e c 0 tal que, se $z \in T$ e $|g(x)-g(z)| \geq \delta|g(z)-g(y)|$ então

$$
\left|g^{\prime}(z)\right| \geq c_{2}\left|g^{\prime}(y)\right|
$$

Prova: Dados $T=(x, y)$ e $J=(v, w)$ temos que

$$
\frac{|g(x)-g(y)|}{|x-y|} \frac{|g(v)-g(w)|}{|v-w|} \geq c_{0} \frac{|g(x)-g(v)|}{|x-v|} \frac{|g(w)-g(y)|}{|w-y|}
$$


ou, denotando por $L, R$ as duas componentes conexas de $T \backslash J$,

$$
\frac{|g(T)|}{|T|} \frac{|g(J)|}{|J|} \geq c_{0} \frac{|g(L)|}{|L|} \frac{|g(R)|}{|R|}
$$

1) Fazendo $v$ tender a $x$ e $w$ tender a $y$ resulta de (3.1) que

$$
\frac{|g(T)|}{|T|} \frac{|g(T)|}{|T|} \geq c_{0} g^{\prime}(x) g^{\prime}(y)
$$

e portanto

$$
\frac{|g(T)|}{|T|} \geq \sqrt{c_{0} g^{\prime}(x) g^{\prime}(y)}
$$

2) Fazendo $v$ e $w$ tenderem a $\alpha$ resulta de (3.1) que

$$
\left|g^{\prime}(\alpha)\right| \geq c_{0} \frac{|g(x)-g(\alpha)|}{|x-\alpha|} \frac{|g(\alpha)-g(y)|}{|\alpha-y|} \frac{|x-y|}{|g(x)-g(y)|} .
$$

Como $g$ é monótona em $(x, y)$,

$$
\frac{|g(x)-g(\alpha)|}{|x-\alpha|} \frac{|x-y|}{|g(x)-g(y)|} \geq 1 \text { ou } \frac{|g(\alpha)-g(y)|}{|\alpha-y|} \frac{|x-y|}{|g(x)-g(y)|} \geq 1 .
$$

Isto implica que

$$
\left|g^{\prime}(\alpha)\right| \geq c_{0} \min \left\{\frac{|g(x)-g(\alpha)|}{|x-\alpha|}, \frac{|g(\alpha)-g(y)|}{|\alpha-y|}\right\} .
$$

Pelo princípio da raiz quadrada,

$$
\left|g^{\prime}(\alpha)\right| \geq c_{0} \min \left\{\sqrt{c_{0} g^{\prime}(x) g^{\prime}(\alpha)}, \sqrt{c_{0} g^{\prime}(\alpha) g^{\prime}(y)}\right\}
$$

e portanto

$$
\left|g^{\prime}(\alpha)\right| \geq c_{0}^{3} \min \left\{g^{\prime}(x), g^{\prime}(y)\right\} .
$$

3) Denotando por $L, R$ as duas componentes conexas de $T \backslash J$ e assumindo $|L| \leq|R|$ temos que

$$
\begin{aligned}
\frac{|J|}{|R|} & \leq \frac{|J|}{|L|}<\frac{|J||T|}{|L||R|} \leq \frac{1}{c_{0}} \frac{|g(T)||g(J)|}{|g(L)||g(R)|} \\
& =\frac{1}{c_{0}} \frac{(|g(L)|+|g(J)|+|g(R)|)|g(J)|}{|g(L)||g(R)|} .
\end{aligned}
$$


Se $|g(R)| \leq|g(L)|$ então temos que

$$
\begin{aligned}
\frac{|J|}{|R|} & <\frac{1}{c_{0}} \frac{|g(J)|}{|g(R)|}\left(\frac{|g(L)|+|g(J)|+|g(R)|}{|g(L)|}\right) \\
& \leq \frac{1}{c_{0} \delta}\left(1+\frac{1}{\delta}+1\right)=\frac{2 \delta+1}{c_{0} \delta^{2}} .
\end{aligned}
$$

Se $|g(L)| \leq|g(R)|$, semelhantemente temos que

$$
\frac{|J|}{|R|} \leq \frac{2 \delta+1}{c_{0} \delta^{2}} .
$$

4) Fazendo $v, w$ tenderem a $z$ resulta de (3.1) que

$$
\begin{aligned}
\left|g^{\prime}(z)\right| & \geq c_{0} \frac{|g(x)-g(z)|}{|g(x)-g(y)|} \frac{|x-y|}{|x-z|} \frac{|g(z)-g(y)|}{|z-y|} \\
& \geq c_{0}\left(\frac{\delta}{1+\delta}\right) \sqrt{c_{0} g^{\prime}(z) g^{\prime}(y)} .
\end{aligned}
$$

E portanto temos que

$$
\left|g^{\prime}(z)\right| \geq c_{0}^{3}\left(\frac{\delta}{1+\delta}\right)^{2}\left|g^{\prime}(y)\right|
$$

O nosso maior interesse é utilizar os Princípios de Köbe no caso em que a função $g$ é uma iterada arbitrária de uma função unimodal restrita a certos intervalos onde esta iterada é monótona. Então temos inicialmente de estimar quanto esta iterada distorce a razão cruzada de três intervalos.

Teorema 3.2.2 Seja $f: I \rightarrow I$ uma função unimodal de classe $C^{2}$ com ponto crítico não-flat. Então existe uma função contínua e limitada $\sigma:[0, \infty] \rightarrow R_{+}$tal que $\sigma(0)=0$ com a seguinte propriedade: se $T$ é um intervalo tal que $\left.f^{m}\right|_{T}: T \rightarrow f^{m}(T)$ é um difeomorfismo, então para todo intervalo $J \subset T \operatorname{com} \bar{J} \subset \operatorname{int}(T)$ verifica-se que

$$
B\left(f^{m}, T, J\right) \geq \exp \left\{-\sigma(\epsilon) \sum_{i=0}^{m-1}\left|f^{i}(T)\right|\right\},
$$


onde $\epsilon=\max \left\{\left|f^{i}(T)\right| ; i=0, \cdots, m-1\right\}$.

Prova: Da definição da razão cruzada, se $\left.f^{m}\right|_{T}$ é monótona e contínua, então

$$
B\left(f^{m}, T, J\right)=\prod_{i=0}^{m-1} B\left(f, f^{i}(T), f^{i}(J)\right) .
$$

De fato,

$$
\begin{aligned}
B\left(f^{m}, T, J\right) & =\frac{D\left(f^{m}(T), f^{m}(J)\right)}{D(T, J)} \\
& =\frac{D\left(f\left(f^{m-1}(T)\right), f\left(f^{m-1}(J)\right)\right)}{D\left(f^{m-1}(T), f^{m-1}(J)\right)} \frac{D\left(f^{m-1}(T), f^{m-1}(J)\right)}{D(T, J)} \\
& =B\left(f, f^{m-1}(T), f^{m-1}(J)\right) B\left(f^{m-1} \cdot T, J\right) .
\end{aligned}
$$

Portanto, basta mostrarmos que existem uma constante $C_{0} \in(0, \infty)$ e uma função continua e crescente, $\sigma:[0, \infty) \rightarrow\left[0, C_{o}\right)$, com $\sigma(0)=0$, tal que

$$
B(f, T, J) \geq \exp \{-\sigma(|T|)|T|\}
$$

para todo intervalo $J \subset T \subset I$ tal que $f^{\prime}(x) \neq 0$ para todo $x \in T$. E é suficiente mostrarmos que para $\xi$ uma outra função

$$
B(f, T, J)-1 \geq-\xi(|T|)|T|,
$$

pois para $x$ em uma pequena vizinhança de 0 temos que $1-x>e^{-x}$. Sejam $V \subset \bar{V} \subset U$ intervalos que contêm o ponto crítico de $f$ e que $f$ é de forma $f(x)=f\left(x_{0}\right)-|\psi(x)|^{l}$ para todo $x \in U$, onde $1<l<\infty$ e $\psi: U \rightarrow(-1,1)$ é $C^{2}$ difeomorfismo.

Caso 1: Assumimos que $T \subset I \backslash V$. Neste caso, sejam $T=[a, d]$ e $J=[b, c]$. Então

$$
\begin{aligned}
B(f, T, J)-1 & =\frac{\frac{f(c)-f(b)}{c-b} \frac{f(d)-f(a)}{d-a}-\frac{f(b)-f(a)}{b-a} \frac{f(d)-f(c)}{d-c}}{\frac{f(b)-f(a)}{b-a} \frac{f(d)-f(c)}{d-c}} \\
& \geq \frac{-1}{k^{2}}\left|\frac{f(c)-f(b)}{c-b} \frac{f(d)-f(a)}{d-a}-\frac{f(b)-f(a)}{b-a} \frac{f(d)-f(c)}{d-c}\right|
\end{aligned}
$$


onde $K=\inf \{|D f(x)| ; x \in I \backslash V\}$. Escrevendo

$$
f(a+x)=f(a)+\mu(a, x) x
$$

temos

$$
\frac{f(d)-f(a)}{d-a}=\mu(a, d-a)
$$

e

$$
\frac{f(b)-f(a)}{b-a}=\mu(a, b-a) .
$$

Também

$$
\begin{aligned}
f(c)-f(b) & =\mu(a, c-a)(c-a)-\mu(a, b-a)(-c+b-a+c) \\
& =[\mu(a, c-a)-\mu(a, b-a)](c-a)+\mu(a, b-a)(c-b) .
\end{aligned}
$$

Portanto

$$
\frac{f(c)-f(b)}{c-b}=\mu(a, b-a)+\frac{\mu(a, c-a)-\mu(a, b-a)}{c-b}(c-a) .
$$

E semelhantemente

$$
\frac{f(d)-f(c)}{d-c}=\mu(a, d-a)+\frac{u(a, d-a)-\mu(a, c-a)}{d-c}(c-a) .
$$

Deste fato e como $|c-a|<|T|$ resulta que

$$
\begin{aligned}
\frac{B(f, T, J)-1}{|T|} & \geq \frac{-1}{k^{2}}\left\{\mid \mu(a, d-a) \frac{\mu(a, c-a)-\mu(a, b-a)}{c-b}\right. \\
& \left.-\mu(a, b-a) \frac{\mu(a, d-a)-\mu(a, c-a)}{d-c} \mid\right\} .
\end{aligned}
$$

Como $f$ é de classe $C^{2}$,

$$
\begin{aligned}
\xi(t) & =\sup _{|T| \leq t} \mid \mu(a, d-a) \frac{\mu(a, c-a)-\mu(a, b-a)}{c-b} \\
& -\mu(a, b-a) \frac{\mu(a, d-a)-\mu(a, c-a)}{d-c} \mid
\end{aligned}
$$

é uma função contínua, crescente, limitada e $\xi(t)$ tende a zero quando $t$ tende a zero. Disto segue o resultado no caso 1 . 
Caso 2: Assumimos que $T \subset U$ Como $1<l<\infty$ e a aplicação $\psi_{l}(x)=x^{l}$ tem a derivada de Schwarz negativa, $B\left(\psi_{\ell}, \psi(T), \psi(J)\right) \geq 1$. Portanto

$$
B(f, T, J)=B\left(\psi_{l}, \psi(T), \psi(J)\right) B(\psi, T, J) \geq B(\psi, T, J)
$$

Como $\psi$ é de $C^{2}$ e $\inf \{|D \psi(x)| ; x \in T\}>0$ verifica-se do caso 1 que $B(\psi, T, J) \geq$ $1-\xi(|T|)|T|$. Portanto, $B(f, T, J) \geq 1-\xi(|T|)|T|$.

Caso 3: Assumimos que $T$ contenha uma componente de $U \backslash V$ então verifica-se que o comprimento de $|T|$ é distante de zero. Neste caso existe $N$ tal que

$$
\frac{B(f, T, J)-1}{|T|} \geq-N>-\infty
$$

Combinando casos 1,2 e 3 , termina a prova do teorema.

Do teorema acima podemos escrever o Princípio de Köbe para iteradas de funções de classe $C^{2}$ da seguinte maneira:

Corolário 3.2.1 Seja $f: I \rightarrow I$ uma função unimodal de classe $C^{2}$ com ponto crítico $c$ de ordem maior que 1. Dados dois intervalos $J \subset T$ tais que $\left.f^{m}\right|_{T}$ é monótona, se $f^{m}(T)$ é uma $\delta$-vizinhança de $f^{m}(J)$ então verifica-se que

$$
\operatorname{dis}\left(f^{m}, J\right) \leq\left(\frac{\delta+1}{\delta}\right)^{2}(1+\sigma(\epsilon) L)
$$

onde $\epsilon$ e $\sigma$ são como no Teorema 3.2. Q e $L=\sum_{i=0}^{m-1}\left|f^{i}(T)\right|$.

Do Lema 3.1.3 resulta que, se $f$ tem derivada de Schwarz negativa, $B\left(f^{m}, T, J\right)>1$. Conseqüentemente nas condições do Corolário acima temos que $\operatorname{dist}\left(f^{m}, J\right) \leq\left(\frac{\delta+1}{\delta}\right)^{2}$. 


\subsection{Aplicação kneading}

Nesta seção estudamos alguns conceitos que serão úteis posteriormente. Dentre eles o conceito de aplicação kneading, o qual foi introduzido em [HF]. Também abordamos a relação entre a aplicação kneading e a seqüência kneading introduzida anteriormente por Milnor e Thurston em [MT].

Definição 3.3.1 Dada uma função unimodal $f: I \rightarrow I$ definimos a sua torre de Hofbauer como sendo a uniâo $\hat{I} \equiv \bigcup_{n=1}^{l} D_{n}, \quad l \in \mathbf{N} \cup\{\infty\}$. onde $D_{n} \subset I$ sâo intervalos definidos indutivamente por $D_{1}=\left(c, c_{1}\right)$ e, para $n \geq 1$,

$$
D_{n+1}=\left\{\begin{array}{cl}
f\left(D_{n}\right), & \text { se } c \notin \overline{D_{n}} \\
\left(c_{n+1}, c_{1}\right), & \text { se } c \in \overline{D_{n}} .
\end{array}\right.
$$

Definimos também a seqüência $\left\{S_{k}\right\}_{k=0}^{\infty}$ da seguinte mantira: como $D_{1} \ni$ c definimos $S_{0}=1$ e para $k \geq 1$ definimos

$$
S_{k}=\min \left\{n ; \overline{D_{n}} \ni c, \text { onde } n>S_{k-1}\right\} .
$$

Os inteiros positivos $S_{k}$ são chamados tempos de corte de $f$. Note que $c_{n}$ sempre pertence ao bordo de $D_{n}$. Se $c_{n}=c$ então $D_{n+1}=\left(c_{1}, c_{1}\right)=\phi$ e neste caso a torre de Hofbauer possui um número finito de níveis.

Observe que se $S_{k}<n \leq S_{k+1}$ então

$$
D_{S_{k}+1}=\left(c_{S_{k}+1}, c_{1}\right) \text { e } D_{n}=\left(c_{n}, c_{n-S_{k}}\right) \text {. }
$$

Então como $D_{S_{k}+1} \subset\left(c, c_{1}\right)$, aplicando $f^{n-S_{k}-1}$ a $D_{S_{k}+1}$ resulta que

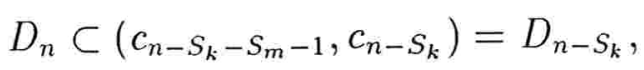

onde $S_{m}<n-S_{k}-1 \leq S_{m+1}$. Também em particular, para $n=S_{k+1}$, temos que $c \in D_{S_{k+1}} \subset D_{S_{k+1}-S_{k}}$ e portanto $S_{k+1}-S_{k}$ também é um tempo de corte. Definimos então a aplicação kneading: $Q: \mathrm{N} \rightarrow \mathrm{N} \cup\{\infty\}$ tal que $Q(0)=0 \mathrm{e}$

$$
S_{Q(k)}=S_{k}-S_{k-1}
$$


para $k \geq 1$. É possível ter $S_{k}=\infty$ e entào $Q(k)=\infty$, neste caso $S(k+j)$ e $Q(k+j)$ não estão definidos para $j \geq 1$.

Para cada $x \neq c$, o ponto simétrico $\tilde{x}$ é definido como sendo o único ponto tal que $x \neq \tilde{x}$ e $f(\tilde{x})=f(x)$. Um ponto $x$ é chamado ponto pré-crítico mais próximo se $f^{n}(x)=c$ para algum $n \geq 1$ e $f^{j}((x, c)) \not \supset c$ para $0<j<n$. Claramente $x$ e $\tilde{x}$ são pontos pré-críticos mais próximos simultaneamente. Se $f$ não possui atrator periódico e nem intervalo errante, então os pontos pré-críticos mais próximos se acumulam em c. Seja $\left\{z_{k}\right\}_{k=0}^{\infty}$ a seqüência dos pontos pré-críticos mais próximos tais que $z_{k}<c$. Então, escrevendo $f^{-1}(c)=\left\{z_{0}, \tilde{z}_{0}\right\}$, temos

$$
z_{0}<\tilde{z}_{1}<z_{2}<\cdots<c<\cdots<\tilde{z}_{2}<\tilde{z}_{1}<\tilde{z}_{0} .
$$

Note que $f\left(\left(z_{0}, c\right)\right)=D_{1}=D_{S_{0}}$. Da definição segue que $S_{1}$ é o menor inteiro positivo tal que $D_{S_{1}} \ni c$. Isto mostra que existe $x \in\left(z_{0}, c\right)$ tal que $f^{S_{1}}(x)=c$ e $f^{i}((x, c)) \not \supset c$ para $i<S_{1}$. Enfim resulta que $x=z_{1}$ e $f^{S_{1}}\left(z_{1}\right)=c$ e indutivamente temose que $f^{S_{k}}\left(z_{k}\right)=c$. Pela definição $\left(z_{k-1}, c\right)$ e $\left(c, \tilde{z}_{k-1}\right)$ são intervalos máximos de monotonicidade de $f^{i}$ para $i \leq S_{k}$. Chamamos $U_{n}$ o intervalo máximo contendo $c$ tal que $\left.f^{n-1}\right|_{f\left(U_{n}\right)}$ é monótona. Então para $S_{k-1}<n \leq S_{k}$ temos que $U_{n}=\left(z_{k-1}, \tilde{z}_{k-1}\right)$. Note que se $n$ é um tempo de corte, então $f^{n}\left(U_{n}\right) \ni c$ e temos que

$$
f^{S_{k}}\left(U_{S_{k}}\right)=\left[c_{S_{k}}, f^{S_{k}}\left(z_{k-1}\right)\right)=\left[c_{S_{k}}, c_{S_{k}-S_{k-1}}\right)=\left[c_{S_{k}}, c_{S_{Q(k)}}\right) .
$$

Seja $A_{k}=\left(z_{k-1}, z_{k}\right) \cup\left(\tilde{z}_{k}, \tilde{z}_{k-1}\right)$ entào como $S_{Q(k)}$ é o maior inteiro tal que $\left.f^{S_{Q(k)}}\right|_{\left(c, c_{S_{k-1}}\right)}$ é monótona, temos que $c_{S_{k-1}} \in \bar{A}_{Q(k)}$. De fato, sabemos que $\left(z_{Q(k)-1}, c\right)$ e $\left(c, \tilde{z}_{Q(k)-1}\right)$ são intervalos máximos de monotonicidade de $f^{S_{Q(k)}}$ e $S_{Q(k)}$ é o maior inteiro tal que $f^{S_{Q(k)}}$ é monótona nestes intervalos. Portanto $\left(c, c_{S_{k-1}}\right) \subset\left(z_{Q(k)-1}, \tilde{z}_{Q(k)-1}\right)$ e se $c_{S_{k-1}} \in$ $\left(z_{Q(k)}, \tilde{z}_{Q(k)}\right)$, temos que $f^{S_{Q(k+1)}}$ é monótona em $\left(c, c_{S_{k-1}}\right)$. Disto resulta que $c_{S_{k-1}} \in$ $\bar{A}_{Q(k)}$.

A seguir introduzimos a seqüência kneading de uma função unimodal e posteriormente estudaremos a relação entre a aplicação kneading e esta sequiência. 
Dado $x \in I$ definimos o seu itinerário $I_{f}(x)=\left(i_{0}, i_{1}, \cdots\right)$, pondo

$$
i_{j}= \begin{cases}0, & \text { se } f^{j}(x)<c \\ 1, & \text { se } f^{j}(x)>c \\ c, & \text { se } f^{j}(x)=c\end{cases}
$$

A seqüência kneading $K_{f}$ é definida como sendo o itinerário de $f(c)$, isto é: $K_{f}=I(f(c))$.

No conjunto $\{0, c, 1\}$ consideramos a relaçào de ordem $<$ dada por $0<c<1$ e no conjunto de todos os itinerários definimos a ordem lexicográfica correspondente, a saber: dados $\mathrm{i}=\left(i_{0}, i_{1}, \cdots\right)$ e $\mathbf{t}=\left(t_{0}, t_{1}, \cdots\right)$ tomamos $n>0$ tal que $i_{j}=t_{j}$ se $0 \leq j<n$ e $i_{n} \neq t_{n}$. E sendo $\tau_{n}(\mathbf{i})$ o número de 1 's entre $i_{0}, i_{1}, \cdots, i_{n-1}$, dizemos que $\mathrm{i} \prec \mathbf{t}$ se

a) $\tau_{n-1}\left(\right.$ i) é par e $i_{n}<t_{n}$

b) $\tau_{n-1}(\mathrm{i})$ é ímpar e $i_{n}>t_{n}$.

Seja $\sum_{3}=\left\{\mathrm{i}=\left(i_{0}, i_{1}, \cdots\right) ; i_{j}=0, c, 1\right\}$. Então a aplicação shift $\sigma: \sum_{3} \rightarrow \sum_{3}$, é dada por

$$
\sigma\left(i_{0}, i_{1}, i_{2}, \cdots\right)=\left(i_{1}, i_{2}, \cdots\right)
$$

Dizemos que um itinerário $\mathrm{v}=\left(\epsilon_{0}, \epsilon_{1}, e_{2}, \cdots\right) \in \sum_{3}$ é admissivel se $\mathrm{v}$ é a seqüência kneading de alguma função unimodal. Um itinerário $\mathrm{v}$ é admissível se e somente se se satisfaz i) $e_{0}=c \quad$ ii) $\sigma^{k}(\mathrm{v}) \prec \sigma(\mathrm{v})$ para todo $k \geq 1$ ou $\mathrm{v}$ é periódico de periodo $n \mathrm{e}$ $\sigma_{k}(\mathrm{v}) \prec \sigma(\mathrm{v})$ para $2 \leq k<n$.

Uma família de funções unimodais $f_{\mu}: I \rightarrow I \operatorname{com} \mu \in[a, b]$ é dita completa se para todo itinerário admissível $\mathrm{v}$ existe $\mu$ tal que $K_{f_{\mu}}=\mathrm{v}$. Sabemos que se $f_{\mu}$ é uma família contínua de funções unimodais de classe $C^{2}$ tal que $K_{f_{a}}=(0,0,0, \cdots)$ e $K_{f_{b}}=$ $(1,0,0, \cdots)$, então $f_{\mu}$ é uma família completa. A família quadrática $q_{a}:[-1,1] \rightarrow[-1,1]$ dada por $q_{a}=-1+a\left(1-x^{2}\right)$ com $a \in(0,2]$ é um exemplo de família completa. Em [CE] mostra-se que se $f$ e $g$ são funçòes S-unimodais tais que $K_{f}=K_{g}$. então $f$ e $g$ são topologicamente conjugadas. 
Dada uma seqüência admissível, $\mathrm{v}=\left(\epsilon_{1}, \epsilon_{2}, \cdots\right)$, definimos uma outra seqüência da seguinte maneira: Tomamos $r_{0}=1$ e definimos $r_{1}$ como sendo o menor inteiro positivo tal que $e_{i}=e_{r_{0}+i}$ para $1 \leq i<r_{1}$ e $\epsilon_{r_{1}} \neq e_{r_{0}+r_{1}}$. Em geral se $R_{k-1}=r_{0}+r_{1}+\cdots+r_{k-1}$, definimos indutivamente $r_{k}$ como sendo o menor inteiro positivo tal que $\epsilon_{i}=e_{R_{k-1}+i}$ para $1 \leq i<r_{k}$ e $\epsilon_{r_{k}} \neq e_{R_{k-1}+r_{k}}$. Entào, para $k \geq 0$ temos que

$$
e_{R_{k}+i}=\epsilon_{i} \text {, para } 1 \leq i<r_{k+1} \text { e } e_{R_{k+1}} \neq \epsilon_{r_{k+1}} \text {. }
$$

É possível termos $r_{k}=\infty$ para algum $k$. Neste caso v é $R_{k-1}$-periódico. Da definição de $r_{k}$ verifica-se que $S_{k}=R_{k}$ e então temos da mesma maneira que $S_{k}-S_{k-1}=S_{Q(k)}$.

Da construção acima sabemos que dada uma seqüência admissivel v, a seqüência $\left\{r_{k}\right\}_{k=1}^{\infty}$ é unicamente construída. E deste resulta temos que $\{Q(k)\}_{k=0}^{\infty}$ é unicamente definida a partir de uma seqüência admissível. Definindo a ordem lexicográfica no conjunto de todas aplicações kneading, a saber: dadas duas aplicaçòes kneading $Q(k)$ e $Q_{1}(k)$ definidas a partir de $\mathbf{v}$ e $\mathbf{v}_{1}$ respectivamente dizemos que $Q(k) \prec Q_{1}(k)$ se existir um inteiro positivo $m$ tal que $Q(k)=Q_{1}(k)$ para $0 \leq k<m$ e $Q(m)>Q_{1}(m)$. Em [HF] prova-se que se $\mathbf{v} \prec \mathbf{v}_{\mathbf{1}}$ então verifica-se que $Q(k) \prec Q_{1}(k)$.

Em $[\mathrm{HF}]$ prova-se também que a condição $\sigma^{k}(\mathrm{v}) \prec \mathrm{v}$ para todo $k \geq 1$, condição de admissibilidade de uma seđ̣:" cia $\mathrm{v}$, é equivalente a seguinte condição: para $k \geq 1 \mathrm{com}$ $Q(k) \neq 0$ existe $m$ tal que

$$
Q(k+j)=Q\left(Q^{2}(k)+j\right) \text { para } 1 \leq j<m \text { e } Q(k+m)>Q\left(Q^{2}(k)+m\right) .
$$

Em [BH-1] prova-se que essa col àr é de fato equivalente à admissibilidade de $\mathrm{v}$.

Lema 3.3.1 Se $f: I \rightarrow I$ é uma $f u$. o unimodal que não possui atrator periódico então $Q(k)<k$, para todo $k \geq 1$.

Prova: Suponha que $Q(k) \geq k$ para algum $k$. Então, como $c_{S_{k-1}} \in \bar{A}_{Q(k)}$ temos que

$$
c_{S_{k-1}} \in\left(z_{Q(k)-1}, \tilde{z}_{Q(k)-1}\right) \subset\left(z_{k-1}, \tilde{z}_{k-1}\right)
$$


Resulta que

$$
f^{S_{k-1}}\left(\left(z_{k-1}, c\right)\right)=f^{S_{k-1}}\left(\left(c, \tilde{z}_{k-1}\right)\right)=\left(c, c_{S_{k-1}}\right) \subset\left(z_{k-1}, \tilde{z}_{k-1}\right) .
$$

Então $f^{S_{k-1}}$ aplica o intervalo $\left(z_{k-1}, c\right)$ ou $\left(c, \tilde{z}_{k-1}\right)$ monotonamente em si mesmo, o que contradiz o fato de $f$ não possuir atrator periódico.

A função unimodal cuja aplicação kneading $Q$ é tal que $Q(k)=k-1$ é a função infinitamente renormalizável de Feigenbaum-Tresser. No caso em que $Q$ é tal que $Q(k)=$ $k-2$ temos a função não-renormalizável de Fibonacci. 


\section{Capítulo 4}

\section{Órbita Crítica da Função Tenda}

A densidade da órbita crítica de uma função tenda $T_{a}$ (isto é. a órbita positiva do valor máximo de $T_{a}$ ) desempenha um importante papel na prova do Teorema $\mathrm{B}$ e é por si só uma questão interessante. Neste capítulo provamos alguns resultados úteis relacionados a esta questão.

\subsection{Aplicação kneading e a órbita crítica}

Lema 4.1.1 Seja $T_{a}: I_{a} \rightarrow I_{a}$ uma funçâo tenda cuja aplicaçâo kneading $Q$ satisfaz $\liminf _{k \rightarrow+\infty} Q(k) \geq 2$, entâo orb $\operatorname{T}_{a}^{+}(c)$ não é densa em $\left[T_{a}^{2}(c), T_{a}(c)\right]$.

Prova: Assumimos que existe $k_{0}$ tal que $Q(k) \geq 2$ para todo $k \geq k_{0}$. Seja $p$ o ponto fixo de $T_{a} \operatorname{com} c<p$. Mostraremos que $p$ não pertence ao fecho de $\operatorname{orb}_{T_{a}}^{+}(c)$. Seja $V$ uma vizinhança de $p$ tal que $c_{i} \notin V$ para $0 \leq i \leq S_{k_{0}}$. Seja $v$ o menor inteiro tal que $T_{a}^{v}(V) \ni c$. Então existe $c_{-v} \in V$, tal que $T_{a}^{v}\left(c_{-v}\right)=c$ e $T_{a}^{i}\left(\left(c_{-v}, p\right)\right) \not \supset c$, para $0 \leq i<v$. Como $p$ é ponto fixo repulsor existe $c_{-v-j} \in V$ tal que $T_{a}^{v+j}\left(c_{-v-j}\right)=c$ e $c \notin T_{a}^{i}\left(\left(c_{-v-j}, p\right)\right)$ para todo $j \geq 0$ e $0 \leq i<v+j$. Se assumirmos que $p<c_{-v}$, então temos que

$$
c_{-v^{\prime-1}}<c_{-v^{\prime-3}}<\cdots<p<\cdots<c_{-v^{-4}}<c_{-v^{\prime}-2}<c_{-v}
$$


Supondo por absurdo que $p \in \overline{\operatorname{orb}_{T_{a}}^{+}(c)}$, entào existe o menor inteiro positivo $n$ tal que $c_{n} \in\left(c_{-v-3}, c_{-\imath^{\prime}-2}\right)$ e temos dois casos:

Caso 1: Existe o menor $\omega \geq 0$ tal que $c_{-\omega}$ e $c_{-\omega-2}$ pertencem ao intervalo $T_{a}^{n}\left(U_{n}\right)$. Entào temos que $T_{a}^{\omega+n}\left(U_{n}\right)$ contém uma componente conexa de $\left(z_{1}, \tilde{z}_{1}\right) \backslash\{c\}$ e que $n+\omega$ é um tempo de corte pois $z_{l} \in U_{n}$ e $T_{a}^{n}\left(z_{l}\right)=c_{-\omega}$. Da minimalidade de $n$ temos que $c_{n+\omega} \notin\left(z_{1}, \tilde{z}_{1}\right)$. Pondo $S_{l}=n+\omega$ temos que $T_{a}^{S_{1}}\left(\left(c, c_{S_{l}}\right)\right) \ni c$ e que $S_{Q(l+1)} \leq S_{1}$, disto resulta que $Q(l+1) \leq 1$. Note que, da definição de $n, S_{l+1}>n+\omega>S_{k_{0}}$ e então $Q(l+1) \geq 2$.

Caso 2: Não existe $\omega \geq 0$ tal que $c_{-\omega}$ e $c_{-\omega-2}$ perteçam ao intervalo $T_{a}^{n}\left(U_{n}\right)$. Da minimalidade de $n$ podemos supor que $c_{n} \in\left(c_{-v^{\prime}-4}, c_{-\ell^{\prime}-2}\right)$ e supondo $S_{k-1}<n \leq S_{k}$ temos que

$$
c_{-v-2} \in T_{a}^{n}\left(U_{n}\right)=\left[c_{n}, c_{m}\right) \subset\left(c_{-v-4}, c_{-v}\right),
$$

onde $m=n-S_{k-1}$, e $T_{a}^{m}\left(U_{m}\right) \subset\left(c_{-v-2}, c_{-v}\right)$. Sendo $\left.T_{a}^{v^{\prime+2}}\right|_{T_{a}^{n}\left(U_{n}\right)}$ monótona e $T_{a}^{v+2+n}\left(U_{n}\right) \ni$ $c$, temos que $n+v+2$ é um tempo de corte $S_{k}$ e $m+v+2=S_{Q(k)}$. Por outro lado, $T_{a}^{v+2+m}\left(U_{m}\right)$ não contém o ponto crítico $c$. Isto contradiz o fato de $v+m+2$ ser um tempo de corte. Resulta que $p \notin \overline{\operatorname{orb}_{T_{a}}^{+}(c)}$.

Então do Lema 4.1.1 segue que se a órbita crítica de $T_{a}$ é densa em $\left[T_{a}^{2}(c), T_{a}(c)\right]$ tem-se que $\liminf _{k \rightarrow+\infty} Q(k) \leq 1$. No próximo capítulo mostraremos que se $\liminf _{k \rightarrow+\infty} Q(k) \leq$ 1 então existe uma condição topológica que proibe a existência de conjugação quasesimétrica entre $T_{a}$ e uma função unimodal de classe $C^{2}$. Neste capítulo mostramos que a densidade da órbita crítica de $T_{a}$ ocorre para quase todo parâmetro $a \in(\sqrt{2}, 2]$.

Lema 4.1.2 Seja $a \in(\sqrt{2}, 2]$ e $J \subset I_{a}$ um intervalo. Então existe $n \in \mathbf{N}$ tal que $\left[T_{a}^{2}(c), T_{a}(c)\right] \subset T_{a}^{n}(J)$.

Prova: Para cada inteiro positivo $i$ temos que

$$
\left|T_{a}^{i+1}(J)\right| \geq \frac{a^{2}}{2}\left|T_{a}^{i-1}(J)\right| \text { ou } T_{a}^{i+1}(J) \supset\left[T_{a}^{2}(c), T_{a}(c)\right] .
$$


De fato, para um intervalo $K^{\prime}$ temos que $\left|T_{a}\left(K^{\prime}\right)\right|=a\left|K^{\prime}\right|$ se $c \notin K^{\prime}$ ou $\left|T_{a}\left(K^{\prime}\right)\right| \geq \frac{a}{2}\left|K^{\prime}\right|$ se $c \in K^{\prime}$. Portanto se $c \notin K^{\circ} \cap T_{a}\left(K^{\prime}\right)$ temos que $\left|T_{a}^{2}\left(K^{\prime}\right)\right| \geq \frac{a^{2}}{2}\left|K^{\prime}\right|$ e se $c \in K^{\prime} \cap T_{a}\left(K^{\prime}\right)$ temos que $\left[c, T_{a}(c)\right] \subset T_{a}(K)$ e então $\left[T_{a}^{2}(c), T_{a}(c)\right] \subset T_{a}^{2}\left(K^{\prime}\right)$. Entretanto, como $a>\sqrt{2}$ o primeiro caso não pode acontecer para todo $i$ e o Lema segue a partir deste fato.

\subsection{Dependência do parâmetro}

Para provarmos o Teorema A investigamos primeiramente as funções $\varphi_{n}:[\sqrt{2}, 2] \rightarrow$ $\left[T_{a}^{2}(c), T_{a}(c)\right]$ definidas por $\varphi_{n}(a)=T_{a}^{n}(c)$, para todo $n \geq 0$. Como $T_{a}(x)=1-a|x|$ temos que

$$
\varphi_{n+1}(a)=1-a\left|\varphi_{n}(a)\right|
$$

Em particular

$$
\varphi_{1}(a)=1, \varphi_{2}(a)=1-a, \varphi_{3}(a)=-a^{2}+a+1, \text { etc. }
$$

Em geral $\varphi_{n}$ é um polinômio quando restrita aos seus intervalos de monotonicidade e é diferenciável em $a$, quando $\varphi_{i}(a) \neq 0$ para todo $1 \leq i<n$. Se $\varphi_{n}(a)>0$ então como $\varphi_{n+1}(a)=1-a \varphi_{n}(a)$ temos que $\varphi_{n+1}^{\prime}(a)=-\varphi_{n}(a)-a \varphi_{n}^{\prime}(a)$. E se $\varphi_{n}(a)<0$ então, como $\varphi_{n+1}(a)=1+a \varphi_{n}(a)$ temos que $\varphi_{n+1}^{\prime}(a)=\varphi_{n}(a)+a \varphi_{n}^{\prime}(a)$. Disto resulta que

$$
\left|\varphi_{n+1}^{\prime}(a)\right|=\left|\varphi_{n}(a)+a \varphi_{n}^{\prime}(a)\right|
$$

E como $\left|\varphi_{n}(a)\right| \leq 1$ temos que

$$
a\left|\varphi_{n}^{\prime}(a)\right|-1 \leq\left|\varphi_{n+1}^{\prime}(a)\right| \leq a\left|\varphi_{n}^{\prime}(a)\right|+1 .
$$

Nosso objetivo é provar que para quase todo $a$ em $[\sqrt{2}, 2]$ o conjunto $\left\{\varphi_{n}(a) ; n=\right.$ $1,2, \ldots\}$ que coincide com $\operatorname{orb}_{T_{a}}^{+}(c)$ é denso em $\left[T_{a}^{2}(c), T_{a}(c)\right]$. 
Para provar o Teorema A são necessários três ingredientes: os primeiros dois são o crescimento exponencial com $n$ do declive de $\varphi_{n}$ e o controle da distorção de $\varphi_{n}$ restrita aos seus intervalos de monotonicidade.

Proposição 4.2.1 Existem constantes positivas $\alpha$ e $\beta$ tais que para todo $n \geq 2$ e $a \in$ $[\sqrt{2}, 2]$ verifica-se que $\alpha a^{n} \leq\left|\varphi_{n}^{\prime}(a)\right| \leq \beta a^{n}$.

Prova: Inicialmente provamos por indução que para todo $n \geq 2$ e $a \in[\sqrt{2}, 2]$ verifica-se que

$$
\left|\varphi_{n}^{\prime}(a)\right| \leq \frac{a^{n-1}-1}{a-1} .
$$

Temos $\varphi_{2}(a)=1-a$ e então $\left|\varphi_{2}^{\prime}(a)\right|=1$. Portanto (4.4) é satisfeita para $n=2$. Assumindo que (4.4) é satisfeita para algum $n$ e usando (4.3) concluimos que

$$
\left|\varphi_{n+1}^{\prime}(a)\right| \leq a\left|\varphi_{n}^{\prime}(a)\right|+1 \leq \frac{a^{n}-1}{a-1} .
$$

Então como $a \geq \sqrt{2}$, para todo $n \geq 2$, temos que

$$
\left|\varphi_{n}^{\prime}(a)\right| \leq \frac{a^{n}}{\sqrt{2}-1} .
$$

Então tomamos $\beta=1 /(\sqrt{2}-1)$. Isto prova uma das desigualdades.

Para provarmos a outra desigualdade observamos inicialmente que

$$
\begin{aligned}
& \left|\varphi_{2}^{\prime}(a)\right|=1>0 \\
& \left|\varphi_{3}^{\prime}(a)\right|=2 a-1>2 \sqrt{2}-1>0 \\
& \left|\varphi_{4}^{\prime}(a)\right|=3 a^{2}-2 a-1 \geq 5-2 \sqrt{2}>0
\end{aligned}
$$

e temos que se a seguinte desigualdade

$$
\left|\varphi_{n}^{\prime}(a)\right| \geq \alpha a^{n}+\frac{1}{a-1}
$$

se verifica para $n$ então a mesma se verifica também para $n+1$. De fato usando (4.3) temos que

$$
\left|\varphi_{n+1}^{\prime}(a)\right| \geq a\left|\varphi_{n}^{\prime}(a)\right|-1 \geq \alpha a^{n+1}+\frac{1}{a-1}
$$


Então para provar a Proposição mostramos (4.5) para $a \in[(\sqrt{5}+1) / 2,2] \operatorname{com} n=3$ e para $a \in[\sqrt{2},(\sqrt{5}+1) / 2] \operatorname{com} n=5$. A constante $\alpha$ que obtemos nestes dois casos podem ser diferentes. Ainda mais, as constantes obtidas nos casos $n=2,3,4$ também podem ser diferentes. No entanto podemos escolher a como sendo a menor destas constantes. Note que para mostrar a existência de $a>0$ tal que (4.5) se verifica para todo $a$ em um intervalo $I$, basta mostrarmos que

$$
\inf _{a \in I}\left[\left|\varphi_{n}^{\prime}(a)\right|-\frac{1}{a-1}\right]>0 .
$$

Como $\left|\varphi_{3}^{\prime}(a)\right|=2 a-1$ para $I=[(\sqrt{5}+1) / 2,2]$ temos que

$$
\begin{aligned}
\inf _{a \in I}\left[\left|\varphi_{3}^{\prime}(a)\right|-\frac{1}{a-1}\right] & =\inf _{a \in I}\left[2 a-1-\frac{1}{a-1}\right] \\
& =\inf _{a \in I} \frac{(2 a-3) a}{a-1}>0 .
\end{aligned}
$$

Isto prova (4.6) para $I=[(\sqrt{5}+1) / 2,2]$.

Para $a \in[\sqrt{2},(\sqrt{5}+1) / 2]$ verifica-se que $\varphi_{5}=1-a+a^{2}+a^{3}-a^{4}$ e como $\varphi_{5}^{\prime}(\sqrt{2})=5-$ $6 \sqrt{2}<0$ e $\varphi_{5}^{\prime \prime}(a)<0$ resulta que $\left|\varphi_{5}^{\prime}(a)\right|$ é crescente. Então como $1 /(a-1)$ é decrescente neste intervalo, para mostrar (4.6) basta mostrarmos que $\left|\varphi_{5}^{\prime}(\sqrt{2})\right|-1 /(\sqrt{2}-1)>0$. De fato

$$
\left|\varphi_{5}^{\prime}(\sqrt{2})\right|(\sqrt{2}-1)-1=(6 \sqrt{2}-5)(\sqrt{2}-1)-1>0
$$

e isto completa a prova.

Portanto de (4.1) o conjunto dos extremos locais de $\varphi_{n+1}$ é a união dos extremos locais de $\varphi_{n}$ com o conjunto de zeros de $\varphi_{n}$. E claramente, em todo intervalo de monotonicidade de $\varphi_{n}$ existe no máximo um zero de $\varphi_{n}$. Precisamos de uma estimativa para a segunda derivada de $\varphi_{n}$.

Lema 4.2.1 Existe uma constante $\gamma>0$ tal que $\left|\varphi_{n}^{\prime \prime}(a)\right| \leq n \gamma a^{n}$ para todo $n \geq 1$ e a $\in$ $[\sqrt{2}, 2]$. 
Prova: Procederemos por induçào. Para $n=1$ temos que $\varphi_{1}(a)=1$ e portanto o Lema é verdadeiro neste caso, independentemente de $\gamma>0$. Suponhamos que o Lema valha para algum $n$. Então se $\beta>0$ é a constante dada pela Proposição 4.2.1 escolhemos $\gamma \geq \sqrt{2} \beta$ e temos que

$$
\begin{aligned}
\left|\varphi_{n+1}^{\prime \prime}(a)\right| & =\left|2 \varphi_{n}^{\prime}(a)+a \varphi_{n}^{\prime \prime}(a)\right| \leq 2\left|\varphi_{n}^{\prime}(a)\right|+a\left|\varphi_{n}^{\prime \prime}(a)\right| \\
& \leq 2 \beta a^{n}+n \gamma a^{n+1} \leq \sqrt{2} \gamma a^{n}+n \gamma a^{n+1} \\
& \leq \gamma a^{n+1}+n \gamma a^{n+1}=(n+1) \gamma a^{n+1}
\end{aligned}
$$

e o Lema segue-se.

Proposição 4.2.2 Para todo $\epsilon>0 \epsilon a_{0}, a_{1} \operatorname{com} \sqrt{2} \leq a_{1}<a_{0}<2$, existe $n_{0}$ tal que para todo $n \geq n_{0}$ e $a \in\left[a_{0}, 2\right]$ verifica-se que

$$
\frac{\left|\varphi_{n+1}^{\prime}(a)\right|}{\left|\varphi_{n}^{\prime}(a)\right|} \geq a_{1}
$$

$e$

$$
\frac{\left|\varphi_{n}^{\prime}(b)\right|}{\left|\varphi_{n}^{\prime}(a)\right|} \leq 1+\epsilon
$$

quando a e b pertencem ao mesmo intervalo de monotonicidade de $\left.\varphi_{n}\right|_{\left[a_{0}, 2\right]}$.

Prova: De (4.3) e da Proposição 4.2.1 temos, para $n \geq 2$ e $a \geq a_{0}$, que

$$
\frac{\left|\varphi_{n+1}^{\prime}(a)\right|}{\left|\varphi_{n}^{\prime}(a)\right|} \geq \frac{a\left|\varphi_{n}^{\prime}(a)\right|-1}{\left|\varphi_{n}^{\prime}(a)\right|} \geq a-\frac{1}{\left|\varphi_{n}^{\prime}(a)\right|} \geq a_{0}-\frac{1}{\alpha a_{0}^{n}} .
$$

Se $n$ é suficientemente grande, então $a_{0}-1 /\left(\alpha a_{0}^{n}\right) \geq a_{1}$ e (4.7) é satisfeita para todo $a \in\left[a_{0}, 2\right]$.

Seja $I=[a, b] \subset\left[a_{0}, 2\right]$ um intervalo tal que $\left.\varphi_{n}\right|_{I}$ é monótona. Então do Lema 4.2.1 temos que

$$
\left|\varphi_{n}^{\prime}(b)\right|-\left|\varphi_{n}^{\prime}(a)\right| \leq|b-a| \sup _{t \in I}\left|\varphi_{n}^{\prime \prime}(t)\right| \leq|b-a| n \gamma 2^{n} .
$$


Como $\left|\varphi_{n}(b)-\varphi_{n}(a)\right| \leq 2$, segue a partir da Proposição 4.2.1 que

$$
|b-a| \leq \frac{\left|\varphi_{n}(b)-\varphi_{n}(a)\right|}{\inf _{t \in I}\left|\varphi_{n}^{\prime}(t)\right|} \leq \frac{2}{\alpha a_{0}^{n}} .
$$

Então concluimos que

$$
\left|\varphi_{n}^{\prime}(b)\right|-\left|\varphi_{n}^{\prime}(a)\right| \leq \frac{2 n \gamma}{\alpha}\left(\frac{2}{a_{0}}\right)^{n}
$$

e usando a Proposiçào 4.2.1 novamente temos que

$$
\frac{\left|\varphi_{n}^{\prime}(b)\right|}{\left|\varphi_{n}^{\prime}(a)\right|}-1 \leq \frac{2 n \gamma}{\alpha}\left(\frac{2}{a_{0}}\right)^{n} \frac{1}{\alpha a_{0}^{n}}=\frac{2 n \gamma}{\alpha^{2}}\left(\frac{2}{a_{0}^{2}}\right)^{n} .
$$

Como $a_{0}^{2}>2$ o Lema segue-se facilmente.

Da Proposição acima segue-se imediatamente um Corolário.

Corolário 4.2.1 Dado $\epsilon>0$ existe $N \in \mathrm{N}$ tal que para todo $n \geq N$ e $U \subset[\sqrt{2}, 2]$, onde $\left.\varphi_{n}\right|_{U}$ é monótona, verifica-se que $\operatorname{dist}\left(\varphi_{n}, U\right) \leq 1+\epsilon$.

Lema 4.2.2 Dado $a \in(\sqrt{2}, 2]$ se $n=S_{k}(a)$ é um tempo de corte de $T_{a}$ então existe um intervalo $J \ni$ a tal que $\left.\varphi_{n}\right|_{J}$ é monótona e $\varphi_{n}(J) \ni c$.

Prova: Consideramos o intervalo $J \ni$ a tal que $\left.\varphi_{n}\right|_{J}$ é monótona e $\mathbf{v}_{a}=\left(\epsilon_{1} \cdots e_{S_{k}} \cdots\right)$ a seqüência kneading de $T_{a}$. Como $n=S_{k}(a)$ é um tempo de corte temos que $T_{a}^{n}\left(U_{n}\right) \ni$ $c(a)$ e existem pontos em $U_{n}$, cujos itinerários correspondem a $\mathbf{v}_{a}$ até $S_{k}(a)$ iteraçôes. Em particular, o itinerário de $c_{-S_{k}(a)}$ é $\left(\epsilon_{1} \cdots e_{S_{k}(a)-1} c e_{1} \cdots\right)$. Pela continuidade de $T_{a}$ em relação ao parâmetro $a$ existe $b \in(\sqrt{2}, 2]$ tal que sua seqüência kneading é periódica: $\mathrm{v}_{b}=\left(e_{1} \cdots e_{S_{k}-1} c e_{1} \cdots\right)$. Disto resulta que $b \in J$ e $\varphi_{n}(b)=c$. 


\subsection{Intervalos de monotonicidade}

O terceiro ingrediente da prova do teorema A depende de estimativas dos comprimentos das imagens dos intervalos de monotonicidade de $\varphi_{n}$. Nesta seção mostramos que estes comprimentos estão uniformemente distantes de zero para quase todo parâmetro $a$ em $[\sqrt{2}, 2]$.

Um intervalo fechado $I \subset[\sqrt{2}, 2]$ será chamado $k$-útil, se $\varphi_{k}$ é monótona em $I$ e uma das extremidades de $\varphi_{k}(I)$ é $c$. Se $I$ é $k$-útil para algum $k$ diremos simplesmente que $I$ é útil. Neste caso poderemos ter vários $k$ 's tal que $I$ seja $k$-útil. Então definimos a ordem de $I$ como sendo o maior $k$ tal que $I$ é $k$-útil.

Para um intervalo $J$ e um inteiro positivo $k$ tal que $\varphi_{k}$ é monótona em $J$, podemos praticar a útil divisão. Pela Proposição 4.2.1 existe $n>0$ tal que $\left.\varphi_{k+n}\right|_{J}$ nào é monótona. Portanto existe o menor $n$ tal que para um ponto $t$ no interior de $J$ tem-se que $\varphi_{k+n}(t)=$ c. Então $\left.\varphi_{k+n}\right|_{J}$ é monótona e $t$ divide $J$ em dois subintervalos $J_{1}$ e $J_{2}$, ambos $k+n$-úteis. Note que se $J$ é $k$-útil, então $n>0$.

Definição 4.3.1 Para $n_{0}$ como na Proposição 4.2.2 seja $N>n_{0}$ um inteiro positivo. Chamamos um intervalo fechado $J$ maduro se existir $n \geq N$ tal que $J$ é $n$-útil $\epsilon$

$$
\varphi_{n}(t)=\varphi_{j}(t) \text { para algum } j \in\{1,2,3\} \text { e algum } t \in J
$$

Caso contrário chamamos imaturo.

Dado um intervalo $I$ construimos por indução uma seqüência $\left\{\mathcal{T}_{n}\right\}_{n=1}^{\infty}$. Definimos $\mathcal{T}_{1}=\{I\}$ e dado $\mathcal{T}_{n}$ se $J \in \mathcal{T}_{n}$ então existem duas possibilidades. Se $J$ é maduro, definimos $\sigma(J)=\{J\}$ e se $J$ é imaturo realizamos uma vez a útil divisão e temos dois subintervalos $J_{1}$ e $J_{2}$. Então definimos $\sigma(J)=\left\{J_{1}, J_{2}\right\}$. E daí definimos $\mathcal{T}_{i+1}=\bigcup_{J \in \mathcal{T}_{i}} \sigma(J)$. 
Lema 4.3.1 Dado $\epsilon>0$ sejam $a_{1}<a_{0}<2$. Sejam $N>n_{0}$ e $I \subset\left[a_{0}, 2\right]$ um intervalo fechado onde $\varphi_{N}$ é monótona. Se $a_{1}^{4}>2(2+\epsilon)$ então para quase todo $a \in I$ existe um intervalo maduro $J$ contendo a. Ainda mais, se $a_{1}^{3}>2(2+\epsilon)$, entâo verifica-se que

$$
\varphi_{n}(t)=\varphi_{j}(t) \text { para algum } j \in\{1,2\} \text { e algum } t \in J
$$

Prova: Seja $B$ o conjunto de todos os pontos de $I$ que não pertencem a intervalos maduros. Provaremos que $\lambda(B)=0$. Suponhamos por absurdo que isto não ocorre.

Se $a \in B \cap I$ então existe uma seqüência de intervalos imaturos $\left\{J_{i}\right\}_{i=1}^{\infty}$ tal que $a \in J_{i} \in \mathcal{T}_{i}$. Então definimos uma seqüência $\left\{\xi_{i}\right\}_{i=1}^{\infty}$ da seguinte mameira:

$$
\xi_{i}(a)=\int_{B \cap J_{i}}\left|\varphi_{k_{i}}^{\prime}\right| d \lambda
$$

onde $k_{i}$ é a ordem de $J_{i}$. Assumindo $a_{1}^{4}>2(2+\epsilon)$ afirmamos que se $J \in \mathcal{T}_{n}$ verifica-se que

$$
\int_{B \cap J} \xi_{n+1} d \lambda \geq \delta \int_{B \cap J} \xi_{n} d \lambda
$$

onde $\delta=\min \left(\sqrt{2}, a_{1}^{4} / 2(2+\epsilon)\right)$.

Se $\lambda(B \cap J)=0$ não temos nada para provar. Assumimos então que $\lambda(B \cap J)>0$, portanto $J$ é imaturo e podemos assumir que

$$
\int_{B \cap J^{1}}\left|\varphi_{k+m}^{\prime}\right| d \lambda \geq \int_{B \cap J^{2}}\left|\varphi_{k+m}^{\prime}\right| d \lambda
$$

onde $m, J^{1}$ e $J^{2}$ são como na definição da útil divisão. Note que as ordens de $J^{i}(i=1,2)$ são maior ou igual a $k+m$. Como $a_{1} \geq \delta$, para $a \in B \cap J$, temos que

$$
\left|\varphi_{k+m}^{\prime}(a)\right| \geq a_{1}^{m}\left|\varphi_{k}^{\prime}(a)\right| \geq \delta\left|\varphi_{k}^{\prime}(a)\right|
$$

Se $B \cap J^{2}$ é vazio então de (4.13), para $a \in B \cap J$, resulta que

$$
\xi_{n+1}(a) \geq \int_{B \cap J^{1}}\left|\varphi_{k+m}^{\prime}\right| d \lambda \geq \delta \int_{B \cap J}\left|\varphi_{k}^{\prime}\right| d \lambda=\delta \xi_{n}(a) .
$$


Portanto (4.11) segue-se.

Assume agora que $B \cap J^{i} \neq \phi$ para $i=1,2$. Então $J^{i}$ são imaturos. Seja $t \in \partial J$ tal que $\varphi_{k}(t)=c$. Lntiano $t \in \partial J^{i}$ para $i=1$ ou 2 e temos que

$$
\varphi_{k+m}(t)=T_{t}^{m}\left(T_{t}^{k}(c)\right)=T_{t}^{m}(c)=\varphi_{m}(t)
$$

Se $m \leq 3$, então pela definição $J^{i}$ satisfaz (4.10) e é maduro o que é uma contradição. Então assumimos que $m \geq 4$, e então de (4.13), para $a \in B \cap J$, temos

$$
\left|\varphi_{k+m}^{\prime}(a)\right| \geq a_{i}^{\prime} \quad{ }^{\prime}(a)|\geq 2(2+\epsilon) \delta| \varphi_{k}^{\prime}(a) \mid .
$$

De (4.8) e (4.12) temos

$$
\frac{\lambda(B \cap J)}{\lambda\left(B \cap J^{1}\right)}=1+\frac{\lambda\left(B \cap J^{2}\right)}{\lambda\left(B \cap J^{1}\right)} \leq 1+\left(1 \quad \frac{+m}{d}|d \lambda|\right.
$$

Deste fato resulta que

$$
\begin{aligned}
\int_{B \cap J^{1}} \xi_{n+1} d \lambda & \geq \lambda\left(B \cap J^{1}\right) \int_{B \cap J^{1}}\left|\varphi_{k+m}^{\prime}\right| d \lambda \\
& \geq \frac{\lambda(B \cap J)}{2+\epsilon} \frac{1}{2} \int_{B \cap J}\left|\varphi_{k+m}^{\prime}\right| d \lambda \geq \delta \lambda(B \cap J) \int_{B \cap J}\left|\varphi_{k}^{\prime}\right| d \lambda \\
& =\delta \int_{B \cap J} \xi_{n} d \lambda .
\end{aligned}
$$

Portanto (4.11) se verifica. Tomamos agora a soma de (4.11) sobre todo $J \in \mathcal{T}_{n}$ e temos

$$
\int_{B} \xi_{n+1} d \lambda \geq \delta \int_{B} \xi_{n} d \lambda
$$

Como $\lambda(B)>0$. ex $\quad \tau_{\text {. tal que }} \lambda(B \cap J)>0$. E como $\xi_{n}>0$ em um subeconjunto de $B$ com medida . 2u. nositiva, temos que $\int_{B} \xi_{n} d \lambda>0$. Como $\delta>1, \int_{B} \xi_{n} d \lambda$ cresce exponencialmentt $\quad$ n. Isso é impossível, pois da definição $\int_{B} \xi_{n} d \lambda \leq 2 \lambda(B)$ para todo $n$. Portanto $\lambda\left(l_{1}=i\right.$.

Para provar o Lema no caso em une $a_{1}^{3}>2(2+\epsilon)$ basta trocarmos na equação (4.14) acima $a_{1}^{4}$ por $a_{1}^{3}$ e na frase anterior " $\leq 3, \cdots \leq 2$ e $m \geq 4$ por $m \geq 3$. 
Agora do Lema 4.3.1 e da definição de intervalo útil temos diretamente o seguinte teorema.

Teorema 4.3.1 Para quase todo a $\in[\sqrt{2}, 2]$ a seguinte propriedade é satisfeita: Para todo $N$ existe $n \geq N$ e pontos $s, t$ no mesmo intervalo de monotonicidade de $\varphi_{n}$ com $a \in(s, t)$, tais que $\varphi_{n}(t)=c e \varphi_{n}(s)=\varphi_{j}(s)$ para algum $j \in\{1,2,3\}$. Ainda mais, para quase todo $a \in[\sqrt[3]{4}, 2]$ se verifica o mesmo com $j \in\{1,2\}$.

Como $\varphi_{1}(a)$ e $\varphi_{2}(a)$ são não-nulos para todo $a \in[\sqrt{2}, 2]$ e $\varphi_{3}(a)$ é não-nulo para $a$ em $[\sqrt{2}, \sqrt[3]{4}]$, temos o seguinte Corolário.

Corolário 4.3.1 Existe $\epsilon>0$ tal que para quase todo $a \in[\sqrt{2}, 2]$ e para todo $N$ existe $n \geq N$ tal que $c \in \varphi_{n}(J)$ e $\lambda\left(\varphi_{n}(J)\right)>\epsilon$, onde $J$ é o intervalo de monotonicidade de $\varphi_{n}$ contendo a

Prova: Do Teorema 4.3.1, existem $t, s$ tais que se $a \in[\sqrt{2}, \sqrt[3]{4}]$ verifica-se que $\varphi_{n}(t)=$ $c$ e $\varphi_{n}(s)=\varphi_{j}(s)$ para algum $j \in\{1,2,3\}$. Se $a \in[\sqrt[3]{4}, 2]$ temos que $\varphi_{n}(t)=c$ e $\varphi_{n}(s)=$ $\varphi_{j}(s)$ para algum $j \in\{1,2\}$. Tomamos $\epsilon<\min \left\{\left|\varphi_{1}(s)-c\right|,\left|\varphi_{2}(s)-c\right|,\left|\varphi_{3}(s)-c\right|\right\} \mathrm{e}$ $J=(s, t)$. 


\section{Capítulo 5}

\section{Conjugação Quase-simétrica}

Uma função unimodal não-renormalizável de classe $C^{2}$ com ponto crítico de ordem $1<l<\infty$ que não possui atrator periódico é topologicamente conjugada a uma função tenda. Neste capítulo exibimos uma condição topológica que proibe a existência de uma conjugação quase-simétrica entre uma função tenda e uma função unimodal de classe $C^{2}$.

\subsection{Uma condição topológica}

Proposição 5.1.1 Seja $f$ uma função unimodal não-renormalizável de classe $C^{2}$ para a qual existe $\vartheta>0$ e uma seqüência $\left\{n_{i}\right\}_{i=1}^{\infty}$ tal que

1. $f^{n_{i}}(c) \rightarrow$ c quando $i \rightarrow \infty$,

2. $f^{n_{i}}\left(U_{n_{i}}\right) \not \ngtr c e$

3. $\left|f^{n_{i}}\left(U_{n_{i}}\right)\right| \geq \vartheta$,

onde $U_{n_{i}}$ é o máximo intervalo contendo c tal que $\left.f^{n_{i}-1}\right|_{f\left(U_{n_{i}}\right)}$ é monótona. Então $f$ é conjugada à uma função tenda $T_{a}$, mas esta conjugaçâo não é quase-simétrica. 
Prova: Suponhamos, por absurdo, que $f$ e $T=T_{a}$ sejam $K$-quase-simetricamente conjugadas. Como $f$ é não-renormalizável temos que $a>\sqrt{2}$ e pelo Lema 2.2.1, existe $M$ tal que $M \geq L(J)$ para todo $J \subset I$. Como $f$ é $C^{2}$ a ordem $l$ do ponto crítico é maior ou igual a 2. Então existem $1<l_{0}<l$ e uma vizinhança $V \ni c$ com a seguinte propriedade: para todo $x \in V$ e todo $y \in(c, x)$ tal que

$$
f(y) \in\left(f(x), \frac{f(c)+f(x)}{2}\right)
$$

temos que

$$
\frac{|x-y|}{|x-c|} \leq \frac{1}{l_{o}} \frac{|f(x)-f(y)|}{|f(x)-f(c)|}
$$

Sejam $B>1$ e $\xi>1$ pequenos tais que $\frac{\xi B}{l_{o}}=r<1$.

Consideramos $V^{\prime \prime} \subset V^{\prime}$ intervalos centralizados em $c$ e $\delta=\frac{\left|V^{\prime}\right| V^{\prime \prime} \mid}{\left|V^{\prime \prime}\right|}$. Dado $J$, um intervalo tal que $\left.f^{n}\right|_{J}$ é monótona e $f^{n}(J) \subset V^{\prime}$, consideramos também $\epsilon:=\max \left\{\left|f^{i}(J)\right| ; 0 \leq\right.$ $i \leq n\}$. Do Corolário 2.1.1 verifica-se que se $\left|V^{\prime}\right|$ tende a zero então $\epsilon$ também tende a zero. Portanto podemos escolher $V^{\prime}$ e $V^{\prime \prime}$ suficientemente pequenos para que $\left(\frac{1+\delta}{\delta}\right)^{2}(1+\sigma(\epsilon) M)$, o que é o controle de distorção correspondente ao Princípio de Köbe Unilateral do caso $C^{2}$, seja menor que $B$.

Definimos duas subseqüências de inteiros positivos $\left\{m_{i}\right\}_{i=1}^{\infty}$ e $\left\{k_{i}\right\}_{i=1}^{\infty}$ da seguinte maneira: definimos $m_{1}=n_{1}$ e $k_{1}$ um inteiro positivo tal que $z_{k_{1}} \in V^{\prime \prime \prime}$. Definimos também $y_{k_{1}}$ tal que

$$
R_{f}\left(k_{1}\right)=\frac{\left|z_{k_{1}}-y_{k_{1}}\right|}{\left|z_{k_{1}}-c\right|} \leq \frac{1}{3 l_{o}} .
$$

Para $i>1$, supondo que $m_{i}$ e $k_{i}$ estejam definidos e satisfaçam

$$
R_{f}\left(k_{i}\right)=\frac{\left|z_{k_{i}}-y_{k_{i}}\right|}{\left|z_{k_{i}}-c\right|} \leq \frac{1}{3 l_{o}}
$$

escolhemos $m_{i+1}$ suficientemente grande de modo que $c_{m_{i+1}} \in\left(\tilde{z}_{k_{i}}, \tilde{z}_{k_{i}}\right) \mathrm{e}$

$$
\frac{\left|z_{k_{i}}-c\right|}{\left|z_{k_{i}}-c_{m_{i+1}}\right|} \leq \xi, \quad \text { se } \quad c_{m_{i+1}} \in\left(z_{k_{i}}, c\right)
$$




$$
\frac{\left|\tilde{z}_{k_{\imath}}-c\right|}{\left|\tilde{z}_{k_{1}}-c_{m_{1+1}}\right|} \leq \xi, \quad \text { se } c_{m_{\imath+1}} \in\left(c, \tilde{z}_{k_{i}}\right) \text {. }
$$
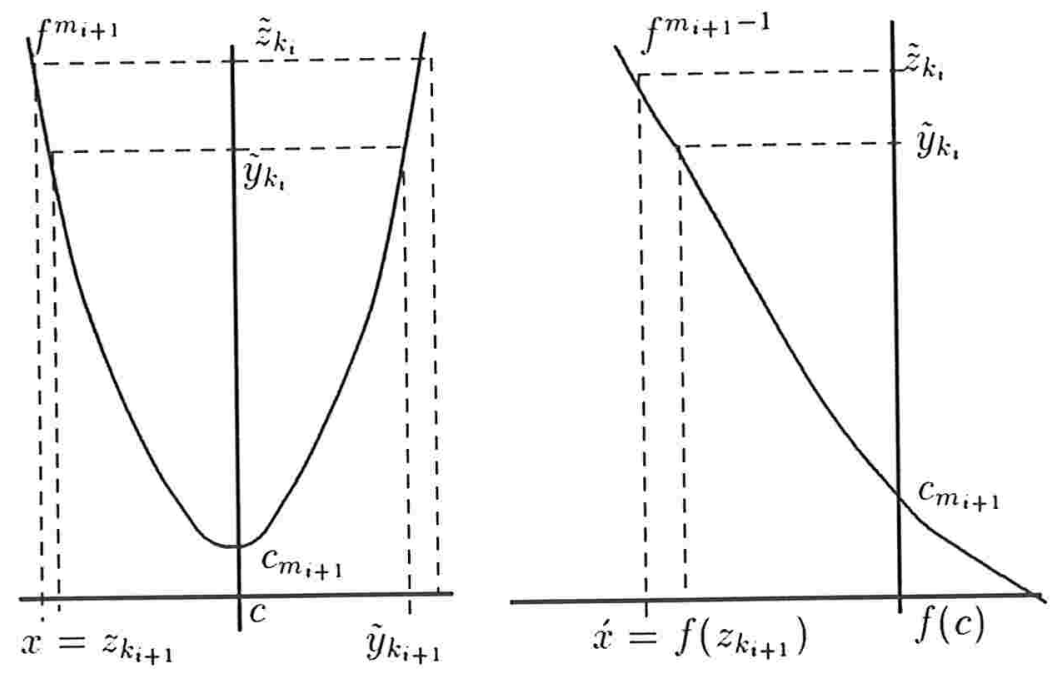

Figura 5.1:

Tomamos $x^{\prime}$ perto de $f(c)$ como sendo $f^{m_{i+1}-1}$-pré-imagem de $z_{k_{i}}$. Então verifica-se que $x^{\prime}$ é a imagem do ponto pré-crítico mais próximo $x$. Seja $k_{i+1}$ tal que $z_{k_{i+1}}=x$. Então temos que $f^{m_{i+1}}\left(z_{k_{i+1}}\right)=z_{k_{i}}$. E definimos $y_{k_{i+1}}$ em $\left(z_{k_{i+1}}, c\right)$ tal que $f^{m_{i+1}}\left(y_{k_{i+1}}\right)=y_{k_{i}}$.

Como $m_{i+1} \in\left\{n_{j}\right\}_{j=1}^{\infty}$ verifica-se que $\left|f^{m_{i+1}}\left(U_{m_{i+1}}\right)\right| \geq \vartheta>\left|V^{\prime \prime}\right|$. Seja $U_{m_{i+1}}^{\prime} \subset$ $(0, c) \cap U_{m_{i+1}}$ o máximo intervalo tal que $f^{m_{i+1}}\left(U_{m_{i+1}}^{\prime}\right) \subset V^{\prime}$. Seja $a \neq c$ outra extremidade de $U_{m_{i+1}}^{\prime}$. Então como $z_{k_{i}} \in V^{\prime \prime}$ verifica-se que, para qualquer $r \in\left(z_{k_{i+1}}, c\right)$, $f^{m_{i+1}-1}((f(a), f(r)))$ é uma $\delta$-vizinhança de $f^{m_{i+1}-1}\left(\left(f\left(z_{k_{i+1}}\right), f(r)\right)\right)$ no lado de $z_{k_{i}}$. Portanto pelo Princípio de Köbe Unilateral temos, em particular, que

$$
\frac{\left|z_{k_{i}}-c_{m_{i+1}}\right|}{\left|f\left(z_{k_{i+1}}\right)-f(c)\right|} \leq B \frac{\left|z_{k_{i}}-y_{k_{i}}\right|}{\left|f\left(z_{k_{i+1}}\right)-f\left(y_{k_{i+1}}\right)\right|} \text {. }
$$

E deste fato resulta que

$$
R_{f}\left(k_{i+1}\right)=\frac{\left|z_{k_{i+1}}-y_{k_{i+1}}\right|}{\left|z_{k_{i+1}}-c\right|} \leq \frac{1}{l_{0}} \frac{\left|f\left(z_{k_{i+1}}\right)-f\left(y_{k_{i+1}}\right)\right|}{\left|f\left(z_{k_{i+1}}\right)-f(c)\right|}
$$




$$
\leq \frac{B}{l_{o}} \frac{\left|z_{k_{i}}-y_{k_{i}}\right|}{\left|z_{k_{i}}-c_{m_{i+1}}\right|} \leq \frac{\xi B}{l_{o}} \frac{\left|z_{k_{i}}-y_{k_{i}}\right|}{\left|z_{k_{i}}-c\right|}=r R_{f}\left(k_{i}\right) .
$$

Como $r<1(5.2)$ se verifica para $k_{i+1}$. Por isso, podemos continuar a indução. Concluimos então que $R_{f}\left(k_{i}\right)$ tende a zero quando $i$ tende a infinito.

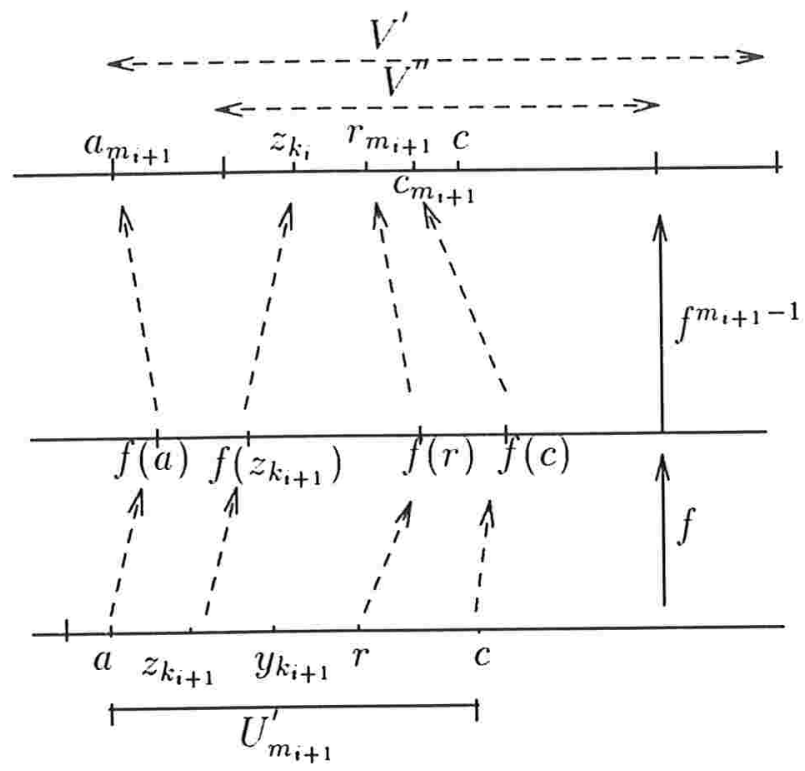

Figura 5.2:

Por outro lado, como $f^{m_{i+1}}$ aplica monotonamente $\left(z_{k_{i+1}}, c\right)$ em $\left(z_{k_{i}}, c\right)$ ou em $\left(c, \tilde{z}_{k_{i}}\right)$, tomando $M_{i}=m_{2}+\cdots+m_{i}$ temos que $T^{M_{i}}$ aplica monotonamente $\left(h\left(z_{i}\right), h(c)\right)$ em $\left(h\left(z_{k_{1}}\right), h(c)\right)$ ou em $\left(h(c), h\left(\tilde{z}_{k_{1}}\right)\right)$ e temos que $T^{M_{i}}\left(h\left(y_{k_{i}}\right)\right)=h\left(y_{k_{1}}\right)$ ou $h\left(\tilde{y}_{k_{1}}\right)$. Como $\left.T^{M_{i}}\right|_{h\left(U_{M_{i}}\right)}$ é linear, para todo $i$, temos que

$$
\begin{aligned}
R_{T}\left(k_{i}\right) & =\frac{\left|h\left(z_{k_{i}}\right)-h\left(y_{k_{i}}\right)\right|}{\left|h\left(z_{k_{i}}\right)-h(c)\right|}=\frac{\left|T^{M_{i}}\left(h\left(z_{k_{i}}\right), h\left(y_{k_{i}}\right)\right)\right|}{\left|T^{M_{i}}\left(h\left(z_{k_{i}}\right), h(c)\right)\right|} \\
& =\frac{\left|h\left(z_{k_{1}}\right)-h\left(y_{k_{1}}\right)\right|}{\left|h\left(z_{k_{1}}\right)-h\left(c_{M_{i}}\right)\right|} \geq \frac{\left|h\left(z_{k_{1}}\right)-h\left(y_{k_{1}}\right)\right|}{\left|h\left(z_{k_{1}}\right)-h(c)\right|}=R_{T}\left(k_{1}\right)>0 .
\end{aligned}
$$

Portanto, pelo Corolário 1.3.1 concluimos que $h$ não pode ser quase-simétrica. 
Seja $\varphi_{n}(a)$ como na seção anterior. Para cada $a \in[\sqrt{2}, 2]$ denotamos a subseqüência dos tempos de corte de $T_{a}$ por $\left\{S_{k}(a)\right\}_{k=0}^{\infty}$. E da mesma maneira denotamos $c_{n}(a)=$ $T_{a}^{n}(c(a))$ e $z_{n}(a) \in T_{a}^{-n}(c(a))$ pré-crítico mais próximo de $c$.

Proposição 5.1.2 Sejam $\epsilon>0 e$

$$
Y(a, \epsilon)=\left(\tilde{z}_{3}(a), z_{3}(a)+\epsilon\right) \cup\left(\tilde{z}_{3}(a)-\epsilon, \tilde{z}_{3}(a)\right) .
$$

Então para quase todo $a \in[\sqrt{2}, 2]$, existe $k \in \mathrm{N}$ tal que $\varphi_{S_{k}(a)}(a) \in Y(a, \epsilon)$.

Prova: Dado $\epsilon \in(0,1)$, pelo Teorema A e pelo Lema 4.1.1 segue que, para quase todo $a \in[\sqrt{2}, 2], \lim _{\inf _{k \rightarrow \infty}} Q_{a}(k) \leq 1$. Seja

$$
A_{\epsilon}=\left\{a \in[\sqrt{2}, 2] ; \liminf _{k \rightarrow \infty} Q_{a}(k) \leq 1 \text { e } T_{a}^{S_{k}(a)}(c) \notin Y(a, \epsilon), k \geq 0\right\}
$$

Seja $N$ um inteiro positivo tal que $\operatorname{dis}\left(\varphi_{n}, J\right) \leq 1+\epsilon / 2$ para todo $n \geq N$ e todo intervalo $J$ tal que $\left.\varphi_{n}\right|_{J}$ é monótona. Pelo Corolário 4.2.1 isto é possível. Suponhamos por absurdo que $\left|A_{\epsilon}\right|>0$, então existe um ponto de densidade $a \in A_{\epsilon}$ e um inteiro suficientemente grande $k$ tal que

1. $S_{k}(a) \geq N$

2. $Q_{a}(k+1) \leq 1$, então $T_{a}^{S_{k}(a)}(c) \notin\left(z_{3}(a), \tilde{z}_{3}(a)\right)$

3. existe uma vizinhança de um lado $J \ni a$, tal que $\varphi_{S_{k}(a)}(J)=\left(c, T_{a}^{S_{k}(a)}(c)\right)$

4. $\frac{\left|J \cap A_{\epsilon}\right|}{|J|} \geq 1-\epsilon / 4$.

De fato, o item 1 segue naturalmente, tomando $k$ suficientemente grande e, para o item 2 , se $T_{a}^{S_{k}(a)}(c) \in\left(z_{3}(a), \tilde{z}_{3}(a)\right)$ então temos que

$$
z_{Q(k+1)}(a) \in T^{S_{k}(a)}\left(\left(z_{k}(a), c\right)\right) \subset\left(z_{3}(a), \tilde{z}_{3}(a)\right)
$$

e resulta que $Q(k+1)>3$ o que é uma contradição. O item 3 segue do Lema 4.2.2. De (4.8) verifica-se que o comprimento máximo do intervalo de monotonicidade de $\varphi_{n}$ tende a zero quando $n$ tende a infinito, disto resulta o item 4 . 
Em particular. $S_{k}(a)=S_{k}$ é constante en $J$. E pela definição $\varphi_{n}(a) \notin Y(a, \epsilon)$ para todo $a \in A_{\epsilon}$ e como a distorção é limitada temos que

$$
\begin{aligned}
1-\frac{\epsilon}{4} & \leq \frac{\left|J \cap A_{\epsilon}\right|}{|J|}=\operatorname{dist}\left(\varphi_{S_{k}}, J\right) \frac{\left|\varphi_{S_{k}}\left(J \cap A_{\epsilon}\right)\right|}{\left|\varphi_{S_{k}}(J)\right|} \\
& \leq\left(1+\frac{\epsilon}{2}\right) \frac{\left|\varphi_{S_{k}}(J) \backslash Y(a, \epsilon)\right|}{\left|\varphi_{S_{k}}(J)\right|} \\
& \leq\left(1+\frac{\epsilon}{2}\right)(1-\epsilon)<1-\frac{\epsilon}{2}
\end{aligned}
$$

o que é uma contradição. Portanto concluimos que $\left|A_{\epsilon}\right|=0$. 


\section{Capítulo 6}

\section{Prova dos Principais Resultados}

\section{Prova do Teorema A:}

Suponhamos que o Teorema é falso, ou seja, existe um conjunto $A$ com medida de Lebesgue positiva tal que para $a \in A$ verifica-se que $\operatorname{orb}_{T_{a}}^{+}(c)$ não é densa em $\left[T_{a}^{2}(c), T_{a}(c)\right]$. Escolhemos então uma base enumerável de abertos em $\mathbf{R}$, ou melhor, definimos o intervalo aberto $B_{n}\left(\frac{p}{q}\right)=\left(\frac{p}{q}-\frac{1}{n}, \frac{p}{q}+\frac{1}{n}\right)$, onde $n, p, q$ são inteiros com $n>0$ e $q \neq 0$. Definimos também o conjunto

$$
A_{n}\left(\frac{p}{q}\right):=\left\{a \in A ; \operatorname{orb}_{T_{a}}^{+}(c) \cap\left(B_{n}\left(\frac{p}{q}\right) \cap\left[T_{a}^{2}(c), T_{a}(c)\right]\right)=\phi\right\} .
$$

Então resulta que $A \subset \cup_{n, p, q \in \mathrm{N}} A_{n}\left(\frac{p}{q}\right)$ e como $|A|>0$ concluimos que existe $n, p, q \in \mathbf{N}$ tal que $\left|A_{n}\left(\frac{p}{q}\right)\right|>0$. Denotamos $K=B_{n}\left(\frac{p}{q}\right) \cap\left[T_{a}^{2}(c), T_{a}(c)\right]$.

Existe um ponto $a \in A_{n}\left(\frac{p}{q}\right)$ que é ponto de densidade de $A_{n}\left(\frac{p}{q}\right)$ e que satisfaz o Corolário 4.3.1. Dado $\epsilon>0$, do Lema 4.1.2 concluimos que existe um intervalo $L \subset$ $[c, c+\epsilon]$ tal que $T_{a}^{k}(L) \subset \operatorname{int}(K)$ para algum $k \geq 1$. De fato, do Lema 4.1.2 existem $n$ e $m$ tais que

$$
T_{a}^{n}(K)=\left[T_{a}^{2}(c), T_{a}(c)\right]=T_{a}^{m}([c, c+\epsilon])
$$

e então $K=T_{a}^{m-n}([c, c+\epsilon])$. Podemos escolher $L \subset[c, c+\epsilon]$ tal que $T_{a}^{m-n}(L) \subset \operatorname{int}(K)$. Pela continuidade de $T_{a}$ em relação ao parâmetro $a$ temos que $T_{b}^{k}(L) \subset K$ para todo $b$ 
de uma vizinhança $U$ de $a$. Se $U$ é suficientemente pequeno. então, como a é ponto de densidade de $A_{n}\left(\frac{p}{q}\right)$, para todo intervalo $J \subset U$, que contém $a$, temos que

$$
\frac{\lambda\left(A_{n}\left(\frac{p}{q}\right) \cap J\right)}{\lambda(J)}>1-\frac{\lambda(L)}{4} .
$$

Pela Proposição 4.2.2, o comprimento máximo do intervalo de monotonicidade de $\varphi_{n}$ tende para 0 quando $n$ tende para infinito. Portanto, se $J$ é o intervalo de monotonicidade de $\varphi_{n}$ contendo $a$ e $n$ é suficientemente grande, então $J \subset U$. Pelo Corolário 4.3.1, podemos obter $n$ arbitrariamente grande tal que ou $[c, c+\epsilon] \subset \varphi_{n}(J)$ ou $[c-\epsilon, c] \subset \varphi_{n}(J)$. Assumimos que $[c, c+\epsilon] \subset \varphi_{n}(J)$; a demonstração para $L \subset[c-\epsilon, c]$ é semelhante, pois $T_{b}^{k}(c-x)=T_{b}^{k}(c+x)$ para $k \geq 1$.

Da Proposição 4.2 .2 para $n$ suficientemente grande temos que

$$
\frac{\lambda(L)}{\lambda(\psi(L))} \leq(1+\epsilon)\left|\varphi_{n}^{\prime}(a)\right| \text { e } \frac{\lambda\left(\varphi_{n}(J)\right)}{\lambda(J)} \geq \frac{\left|\varphi_{n}^{\prime}(a)\right|}{1+\epsilon},
$$

onde $\psi=\left(\left.\varphi\right|_{J}\right)^{-1}$. Portanto temos que

$$
\frac{\lambda(\psi(L))}{\lambda(J)} \geq \frac{\lambda(L)}{\lambda\left(\varphi_{n}(J)\right)} \frac{1}{(1+\epsilon)^{2}} \geq \frac{\lambda(L)}{4} .
$$

Se $b \in \psi(L)$ então $b \in J$ e $\varphi_{n}(b) \in L$. Então como $T_{b}^{k+n}(c) \in K$ temos que $b \in$ $J \backslash A_{n}\left(\frac{p}{q}\right)$. Portanto, $\lambda\left(J \backslash A_{n}\left(\frac{p}{q}\right)\right) / \lambda(J) \geq \lambda(L) / 4$. Isto contradiz (6.1) pois

$$
\frac{\lambda(L)}{4}>1-\frac{\lambda\left(A_{n}\left(\frac{p}{q}\right) \cap J\right)}{\lambda(J)}=\frac{\lambda\left(J \backslash A_{n}\left(\frac{p}{q}\right)\right)}{\lambda(J)}
$$

e o Teorema A segue-se. 


\section{Prova do Teorema B:}

Suponhamos que $a \in[\sqrt{2}, 2]$, da Proposição 5.1 .2 resulta que $A_{\epsilon}$ tem medida de Lebesgue zero, para todo $\epsilon>0$. Então verifica-se que

$$
A=\left\{a \in[\sqrt{2}, 2] ; \liminf _{k \rightarrow \infty} Q_{a}(k) \leq 1\right\} \backslash \bigcup_{i=1}^{\infty} A_{\frac{1}{i}}
$$

tem medida de Lebesgue total em $[\sqrt{2}, 2]$. Tomamos $a \in A$ e então existe uma seqüência $\left\{k_{i}\right\}_{i=1}^{\infty}$ tal que $T_{a}^{S_{k_{i}}}(c) \in Y\left(a, \frac{1}{i}\right)$ para todo $i$. Como $Q(k)<k$ para todo $k$, verifica-se que $S_{3}(a) \leq 8$ e temos que

$$
T_{a}^{S_{k_{i}}(a)+S_{3}(a)}(c) \in\left(c-\frac{2^{8}}{i}, c\right) \text { ou }\left(c, c+\frac{2^{8}}{i}\right) .
$$

Como $T^{j}\left(\left(z_{k_{i}}, c\right)\right) \not \supset c$ para todo $0 \leq j \leq S_{k_{i}}+S_{3}$ resulta que $S_{k_{i}}+S_{3}<S_{k_{i}+1}$ e $U_{S_{k_{i}}+S_{3}}=\left(z_{k_{i}}, \tilde{z}_{k_{i}}\right)$. Deste fato resulta que

$$
T_{a}^{S_{k_{i}}(a)+S_{3}(a)}\left(U_{S_{k_{i}}(a)+S_{3}(a)}\right)=\left(T_{a}^{S_{3}(a)}(c) T_{a}^{S_{k_{i}}(a)+S_{3}(a)}(c)\right) \not \supset c .
$$

Tomando $n_{i}=S_{k_{i}}(a)+S_{3}(a)$ temos que $T_{a}^{n_{i}}\left(U_{n_{i}}\right)$ não contém $c$ e $T_{a}^{n_{i}}(c)$ tende a $c$ quando $i$ tende a infinito. Como $T_{a}^{n_{i}}(c) \rightarrow c$, para todo $i$ temos que $\left|T_{a}^{n_{i}}\left(U_{n_{i}}\right)\right|$ é distante de zero. Portanto a seqüência $\left\{n_{i}\right\}_{i=1}^{\infty}$ satisfaz as três condições da Proposição 5.1.1 e isto prova o Teorema B. 
[MT] Milnor, J., Thurston, W.: On iterated maps of the interval- Lect.Notes Math. 1342, 46.5-56.3 (19S8)

[NP] Nowicki, T., Przytycki, F.: The conjugacy of Collet-Eckmann's map of the interval with the tent map is Hölder continuous- Ergodic Th. Dynam. Sys. 9, 379-388 (1989)

[RD] Roberto, L., Devaney: An Introduction to Chaotic Dynamical Systems second edition- Departament of Mathematics, Boston University (1948)

[SD] Sullivan, D.: Quasiconformal homeomorphisms in dynamics, topology and geometry- Proc. Int. Congress of Matematics-Berkeley (1986)

[SM] Shub, M.: Endomorphisms of campact diferentiable manifolds-Amer. J. Math XCI. 175-199 (1969)

[SN] Sands, D., Nowicki, T.: Quasisimmetric invariance of the Collet-Eckmann condition- Ergodic Th. Dynam. Sys. 18, 703-715 (1998)

[SS] Shub, M., Sullivan, D.: Expanding endomorphisms of the circle revisited- Ergodic Th. Dynam. Sys. 5, 285-289 (1985)

[WR] Walte,r R.: Real and Complex Analysis- Mcgraw-Hill Series in Higher Mathematics (1996)

[YJ] Yoccoz, J.C.: Conjugaison differentiable des difféomorphismes du cercle dont le nombre de rotation vérifie une condition Diophantienne-Ann. Sci. Ec. Norm. Sup. 17, 333-361 (1984) 


\section{Referências Bibliográficas}

[BA] Beurling, A., Ahlfors, L.: The boundary correspondence under quasiconformal mappings- Acta Math. 96, 125-142 (1956)

[BD] Brucks, K., Diamand, D.: Dense Orbits of Critical Points for the Tent Map- Contemporary Math. 117, 57-61 (1991)

[BM] Brucks, K., Misiurewicz, M.: Trajectory of the turning point is dense for almost all tent maps- Ergodic Th. Dynam. Sys. 16, 1173-118:3 (1996)

[BH] Bruin, H.: Quasi-symmetry of conjugacies between interval maps- Nonlinearty 9 1191-1207 (1996)

[BH-1] Bruin, H.: Combinatorics of the Lineading Map- International Journal of Bifurcation and Chaos, 5, No.5 1339-1349 (1995)

[CE] Collet, P., Eckmann, J.P.: Iterated maps on the interval as dynamical systemsProgress in Physics (1948)

[GS] Graczyk, J., Swiatek, G.: Hyperbolicity is dense in the real quadratic family- Penn State preprint (1995)

[HF] Hofbauer, F.: The Topological Entropy of the Transformation $x \rightarrow$ ax $(1-x)$ Monath. Math. 90 117-141 (1980)

[MS] de Melo, W., van Strein, S.: One-dimensional dynamics- Ergebnisse der Mathematic und ihrer Grenzgebiete (Berlin: Springer) (1993) 